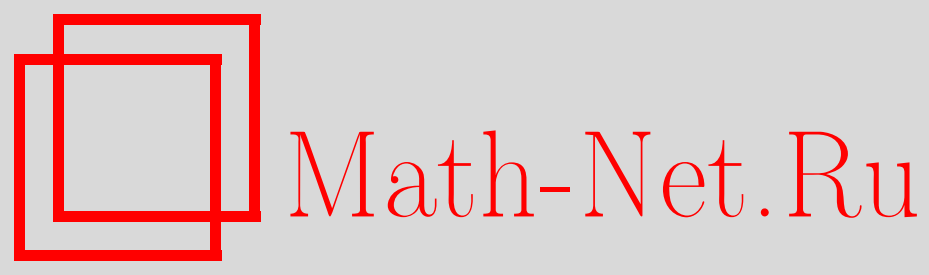

Н. Б. Медведева, Об аналитической неразрешимости проблемы устойчивости на плоскости, УМН, 2013, том 68, выпуск 5, 147-176

DOI: https://doi.org/10.4213/rm9524

Использование Общероссийского математического портала Math-Net.Ru подразумевает, что вы прочитали и согласны с пользовательским соглашением http://www . mathnet.ru/rus/agreement

Параметры загрузки:

IP : 54.198 .187 .58

26 апреля 2023 г., 16:33:02

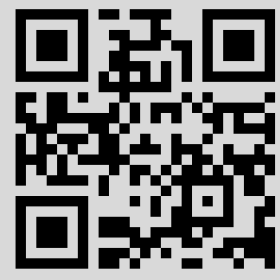




\section{Об аналитической неразрешимости проблемы устойчивости на плоскости}

\section{Н. Б. Медведева}

Дается обзор по вопросам алгебраической и аналитической разрешимости проблемы устойчивости особой точки векторного поля на плоскости, и в частности проблемы различения центра и фокуса. Доказана аналитическая неразрешимость проблемы устойчивости на плоскости.

Библиография: 30 названий.

Ключевые слова: векторное поле, монодромная особая точка, фокус, центр, преобразование монодромии, диаграмма Ньютона, раздутие особенностей.

DOI: $10.4213 / \mathrm{rm} 9524$

\section{СоДЕРЖАНИЕ}

1. Введение......................................... 148

1.1. Алгебраическая и аналитическая разрешимость локальных задач 148

1.2. Монодромные особые точки........................ 150

1.3. Пространство монодромных ростков ................... 152

1.4. Проблема различения центра и фокуса и проблема устойчивости на плоскости ................................. 152

1.5. Диаграмма Ньютона . . . . . . . . . . . . . . . . . . . . . . . . . . 153

1.6. Раздутие по диаграмме Ньютона . . . . . . . . . . . . . . . . . . . . . . . . 155

1.7. Простейшие монодромные классы . . . . . . . . . . . . . . . 158

1.8. Сложные монодромные особые точки.................. 160

1.9. Второй член преобразования монодромии ............... 161

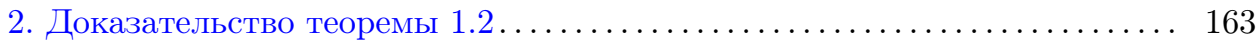

3. Асимптотика границы устойчивости ..................... 167

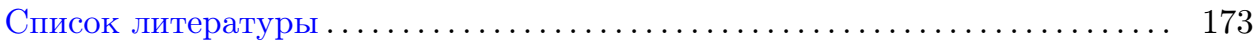

Работа выполнена при поддержке РФФИ (грант № 10-01-00587-а) и ФЦП 02.740.110612. 


\section{1. Введение}

ОПРЕДЕЛЕНИЕ 1.1. Ростком векторного поля в особой точке называется класс эквивалентности векторных полей, совпадающих в некоторой окрестности особой точки. $k$-струей векторного поля в особой точке 0 называется класс ростков векторных полей в точке 0, отличающихся друг от друга на величину $o\left(|x|^{k}\right)$ при $x \rightarrow 0$.

Выбор системы координат в фазовом пространстве превращает пространство $k$-струй в координатное пространство: каждой струе соответствует набор коэффициентов тейлоровского разложения векторного поля до степени $k$.

\section{1. Алгебраическая и аналитическая разрешимость локальных за-} дач. Пусть $W_{0}$ - пространство всех ростков аналитических векторных полей с особой точкой $0 \in \mathbb{R}^{n} ; J_{0}^{k}-$ пространство $k$-струй векторных полей в точке $0, p_{k}: W_{0} \rightarrow J_{0}^{k}$ - естественная проекция. Если $W$ - подмножество $W_{0}$, то через $W^{(k)}$ будем обозначать множество $k$-струй ростков из $W$.

Понятие алгебраически разрешимой локальной задачи введено В.И. Арнольдом в работе [1]. Согласно Арнольду, под задачей понимается разбиение пространства ростков $W_{0}$ на непересекающиеся подмножества: $W_{0}=\bigcup_{i} S_{i}$, где $i \in I, I$ - некоторое множество индексов. Если, например, рассматривается задача об устойчивости по Ляпунову, то таких подмножеств всего два: $S_{1}$, состоящее из устойчивых ростков (т. е. ростков, имеющих устойчивую особую точку), и $S_{2}$, состоящее из неустойчивых ростков.

ОПРЕДЕЛЕНИЕ 1.2. Подмножество вещественного числового пространства называется полуалгебраическим множеством, если оно является объединением конечного числа подмножеств, задаваемых конечным числом алгебраических уравнений и неравенств.

Подмножество пространства ростков называется полуалгебраическим, если оно является объединением конечного числа подмножеств, задаваемых конечным числом алгебраических уравнений и неравенств относительно коэффициентов некоторой струи.

ОПРЕДЕЛЕНИЕ 1.3 [1], [2]. Задача о ростках векторных полей в особой точке $0 \in \mathbb{R}^{n}$ называется алгебраически разрешимой, если для любого $k$ существует разбиение пространства струй $J_{0}^{k}$ на непересекающиеся полуалгебраические подмножества, $J_{0}^{k}=\bigcup_{i \in I} J_{k, i} \cup J_{k}^{\prime}$, такие, что

1) $p_{k}^{-1} J_{k, i} \subset S_{i}, i \in I$;

2) $\lim _{k \rightarrow \infty} \operatorname{codim} J_{k}^{\prime}=\infty$.

Алгебраическая разрешимость задачи означает, что ответ в задаче может быть получен с помощью конечного числа арифметических операций над коэффициентами струи за исключением ростков из некоторого исключительного множества коразмерности бесконечность. 
Например, если речь идет о различении устойчивых и неустойчивых ростков, то $i \in\{1,2\}$ и все ростки, $k$-струи которых принадлежат $J_{k, 1}$, устойчивы, а все ростки, $k$-струи которых принадлежат $J_{k, 2}$, неустойчивы.

Можно говорить об алгебраической разрешимости локальной задачи не в пространстве всех ростков $W_{0}$, а ограничиваясь некоторым его подмножеством $W$. Тогда п. 1) определения 1.3 заменяется условием $W \cap p_{k}^{-1} J_{k, i} \subset S_{i}$.

В случае, когда задача не является алгебраически разрешимой, было предложено ввести понятие аналитической разрешимости (В. И. Арнольд, Ю. С. Ильяшенко [2], [3]). Аналитическая разрешимость задачи означает, что ответ в задаче может быть получен с помощью вычисления конечного числа значений аналитических функций от коэффициентов струи за исключением ростков из некоторого множества бесконечной коразмерности. Отсутствие аналитической разрешимости может привести, например, к таким патологиям, как накопление бесконечного числа связных компонент различных множеств $S_{i}$ в окрестности одной точки пространства ростков.

ОПРЕДЕЛЕНИЕ 1.4. Подмножество $V$ области $D$ конечномерного пространства называется полуаналитическим подмножеством области $D$, если у каждой точки $a \in D$ существует ее окрестность $U$ в области $D$ такая, что пересечение $V \cap U$ является конечным объединением множеств, задаваемых конечным числом уравнений вида $f=0$ и неравенств вида $g>0$, где $f$ и $g$ - аналитические в $U$ функции.

ЗАмЕчАниЕ 1.1. Полуаналитическое подмножество области может не быть полуаналитическим подмножеством всего пространства. Например, множество на плоскости, задаваемое уравнением $y-\sin (1 / x)=0$, является полуаналитическим подмножеством области $x>0$, но не является полуаналитическим подмножеством всей плоскости.

На некотором множестве $W \subset W_{0}$ рассмотрим бинарную локальную задачу, т. е. задачу, в которой решается вопрос о принадлежности ростка к одному из двух классов $S_{1}$ и $S_{2}$.

ОПРЕДЕЛЕНИЕ 1.5 [4]. Бинарная задача называется аналитически разрешимой в классе $W \subset W_{0}$, если для любого $k$ существует такое разбиение некоторой окрестности $U_{k}$ множества $W^{(k)}$ в пространстве $J_{0}^{k}$ на непересекающиеся полуаналитические подмножества этой окрестности, $U_{k}=J_{k, 1} \cup J_{k, 2} \cup J_{k}^{\prime}$, такие, что

1) $W \cap p_{k}^{-1} J_{k, 1} \subset S_{1}, W \cap p_{k}^{-1} J_{k, 2} \subset S_{2}$;

2) $\lim _{k \rightarrow \infty} \operatorname{codim} J_{k}^{\prime}=\infty$.

Множество $J_{k}^{\prime}$ называется множеством нейтралъных $k$-струй.

Если в каждом из каких-либо двух подмножеств пространства ростков задача аналитически разрешима, то в силу замечания 1.1 отсюда вовсе не следует ее аналитическая разрешимость в объединении этих подмножеств.

Обзор результатов, касающихся алгебраической и аналитической разрешимости некоторых локальных задач, приведен в [2], [3]. Например, проблема устойчивости по Ляпунову и проблема топологической классификации особых 
точек аналитических векторных полей $\left(\right.$ в $\left.\mathbb{R}^{n}\right)$ являются не только алгебраически, но и аналитически неразрешимыми [5], [6]. Построенные в [5] и [6] примеры относятся к многомерному случаю $(n>2)$.

В случае $n=2$ существуют хорошо разработанные алгоритмы исследования устойчивости, поэтому до последнего времени существовала надежда, что задача об устойчивости окажется хотя бы аналитически разрешимой. В дальнейшем мы будем рассматривать только векторные поля на вещественной плоскости $(n=2)$.

\section{2. Монодромные особые точки.}

ОПРЕДЕЛЕНиЕ 1.6. Фазовая кривая векторного поля на плоскости называется характеристической траекторией особой точки, если она входит в эту точку при $t \rightarrow+\infty$ или $t \rightarrow-\infty$, касаясь некоторой прямой (см. рис. $1, \mathrm{a})$ ).

Известно [2], что вещественно-изолированная особая точка аналитического векторного поля на плоскости либо имеет характеристическую траекторию, либо монодромна, т. е. каждая траектория в окрестности этой особой точки является топологически либо спиралью, либо окружностью. Для монодромной особой точки определено преобразование монодромии, переводящее некоторую гладкую кривую с началом в особой точке (полутрансверсаль) в себя вдоль траекторий векторного поля. Образ и прообраз при этом отображении лежат на одном отрезке траектории, делающем один оборот вокруг особой точки (см. рис. $1, \mathrm{~b})$ ). Строгое определение монодромной особой точки и преобразования монодромии дано в [2].

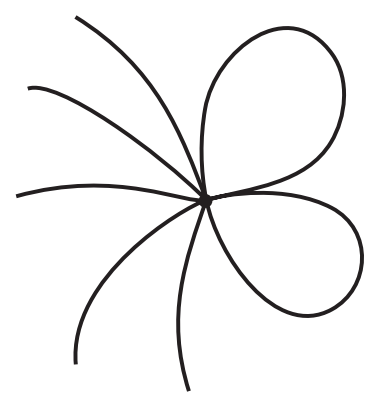

a)

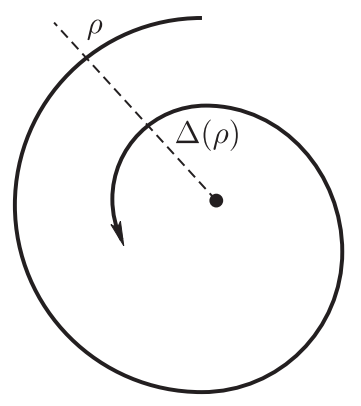

b)

Рис. 1. а) Особая точка с характеристической траекторией. b) Монодромная особая точка. Пунктиром изображена полутрансверсаль, $\rho-$ параметр на полутрансверсали, $\Delta(\rho)$ - преобразование монодромии.

Легко привести пример гладкого векторного поля, особая точка которого не имеет характеристической траектории и не является монодромной [2].

Из теоремы о конечности числа предельных циклов [7] следует, что монодромная особая точка аналитического векторного поля на плоскости является либо центром, либо фокусом. 
Перечислим основные типы монодромных особых точек, для которых хорошо известны алгоритмы различения центра и фокуса.

Наиболее популярный и широко исследованный класс - это ростки, имеющие в особой точке центр по линейным членам. Необходимым и достаточным условием центра [8], [9], [2] в этом случае является существование формального первого интеграла. Условием же существования последнего является обращение в нуль бесконечного числа ляпуновских фокусных величин, которые являются полиномами от тейлоровских коэффициентов векторного поля.

Следующий класс - это ростки, имеющие в особой точке линейную часть в виде ненулевой нильпотентной жордановой клетки. Этот класс впервые был исследован А. М. Ляпуновым [10] с помощью раздутия, использующего специальные функции. Условием центра здесь, как и в случае центра по линейным членам, является обращение в нуль бесконечного числа полиномов от тейлоровских коэффициентов ростка. Совсем другой подход к решению этой проблемы используется в работе [11].

Еще один класс монодромных ростков исследован в [12]. Пусть разложение Тейлора векторного поля в особой точке $(x, y)=(0,0)$ начинается с $r$-х степеней:

$$
\left(X_{r}+\cdots\right) \frac{\partial}{\partial x}+\left(Y_{r}+\cdots\right) \frac{\partial}{\partial y}
$$

где $X_{r}$ и $Y_{r}$ - однородные полиномы степени $r$ и $r$ нечетно. Говорят, что векторное поле не имеет в особой точке исключительных направлений, если однородный многочлен $-y X_{r}+x Y_{r}$ не имеет вещественных линейных делителей. Особые точки без исключительных направлений всегда монодромны. Переход к полярным координатам и последовательное решение уравнений в вариациях позволяют выразить условия центра в виде равенства нулю бесконечного числа интегралов, которые являются аналитическими функциями от тейлоровских коэффициентов векторного поля.

Общим для всех трех перечисленных случаев является следующее. С помощью специальной замены переменных векторное поле, имеющее в нуле монодромную особую точку одного из перечисленных типов, можно превратить в векторное поле, определенное в полуокрестности инвариантной окружности, на которой нет особых точек. Другими словами, исследование векторного поля в окрестности особой точки может быть сведено к исследованию векторного поля в окрестности замкнутой траектории. Преобразование монодромии в этих случаях является аналитической функцией $\Delta(x)=c x+\sum_{k=2}^{\infty} c_{k} x^{k}$, тейлоровские коэффициенты которой можно вычислить путем последовательного решения уравнений в вариациях. Поскольку в случае центра $\Delta(x) \equiv x$, то различение центра и фокуса состоит в сравнении преобразования монодромии с тождественным отображением. Если хотя бы одна из величин $\ln c, c_{2}, c_{3}, \ldots$ отлична от нуля, то особая точка является фокусом. 
1.3. Пространство монодромных ростков. Росток векторного поля в монодромной особой точке будем называть монодромным ростком. Множество всех монодромных ростков обозначим буквой $\mathscr{M}$.

Из теорем о раздутии особенностей (см., например, [13]) следует, что проблема различения монодромных ростков и ростков с характеристической траекторией алгебраически разрешима. Более того, множество $\mathscr{M}$ представляет собой счетное объединение,

$$
\mathscr{M}=\bigcup_{\alpha} M_{\alpha}
$$

полуалгебраических множеств различных коразмерностей, состоящих из монодромных ростков. В [4] предложена классификация монодромных ростков в соответствии с процессом раздутия особенности по диаграммам Ньютона: для всех ростков, принадлежащих одному классу $M_{\alpha}$, процесс раздутия по диаграммам Ньютона осуществляется одинаковым образом. Более подробно классы $M_{\alpha}$ описаны в п. 1.7. Точное определение классов $M_{\alpha}$, названных простейшими монодромными классами, дано в [4]. Примерами простейших монодромных классов являются: 1) множество ростков с невещественными собственными значениями линейной части векторного поля в особой точке, 2) множество ростков с линейной частью в виде нильпотентной жордановой клетки с фиксированной диаграммой Ньютона, 3) множество ростков без исключительных направлений с фиксированной степенью младших членов.

Из теорем о раздутии особенностей вытекает, что проблема устойчивости по Ляпунову и проблема орбитальной топологической классификации на множестве ростков с характеристической траекторией алгебраически разрешима.

\section{4. Проблема различения центра и фокуса и проблема устойчи-} вости на плоскости. Проблему устойчивости в любом классе монодромных ростков будем, отдавая дань традиции, называть проблемой различения иентра и фокуса, хотя в контексте алгебраической и аналитической разрешимости реально имеет место различение между устойчивым и неустойчивым фокусом.

В классе ростков, имеющих центр по линейным членам, а также в классе ростков, имеющих линейную часть в виде нильпотентной жордановой клетки, проблема различения центра и фокуса, а следовательно, и проблема устойчивости алгебраически разрешима [2], [3]. Это следует из алгебраичности условий центра и из доказанного в [4] факта, что множество центров имеет коразмерность $\infty$.

Как было обнаружено Ю.С. Ильяшенко [14], существуют множества $M_{\alpha}$, в которых проблема различения центра и фокуса, а следовательно, и проблема устойчивости не является алгебраически разрешимой. Более точно, неалгебраическая граница устойчивости была обнаружена в некотором семействе, состоящем из ростков без исключительных направлений. Тем самым, проблема различения центра и фокуса не является алгебраически разрешимой на всем множестве монодромных ростков. 
Если под $S_{1}$ и $S_{2}$ в определении аналитической разрешимости понимать множество устойчивых и неустойчивых фокусов соответственно, то имеет место следующая теорема.

ТеОРема 1.1 [4], [15]. Проблема различения центра и фокуса аналитически разрешима в любом простейшем монодромном классе.

Утверждение этой теоремы означает, что множество ростков, имеющих в нуле особую точку типа центр, имеет коразмерность $\infty$, а наличие фокуса (устойчивого или неустойчивого) может быть установлено путем вычисления конечного числа аналитических функций от тейлоровских коэффициентов ростка. Итак, пространство ростков разбито в счетное объединение полуалгебраических множеств (одни из которых состоят из монодромных ростков, а другие из ростков с характеристической траекторией), в каждом из которых проблема устойчивости аналитически разрешима (в некоторых даже алгебраически разрешима). Однако, как отмечалось выше, отсюда еще не следует аналитическая разрешимость проблемы устойчивости во всем пространстве ростков.

Основным результатом настоящей работы является следующая теорема, из которой вытекает аналитическая неразрешимость проблемы устойчивости на плоскости.

ТЕОРема 1.2. Существуют простейший монодромный класс $M$, число $k u$ непустые непересекающиеся полуаналитические подмножества $J_{k}^{1}, J_{k}^{2}, J_{k}^{\prime} \subset$ $M^{(k)}$ mакие, что $M^{(k)}=J_{k}^{1} \cup J_{k}^{2} \cup J_{k}^{\prime}, \operatorname{codim} J_{k}^{\prime}=2$ в $M^{(k)}, p_{k}^{-1} J_{k}^{1} \subset S_{1}$, $p_{k}^{-1} J_{k}^{2} \subset S_{2}$, причем $J_{k}^{\prime}$ не является полуаналитическим подмножсеством пространства струй $J_{0}^{k}$.

1.5. Диаграмма Ньютона. Рассмотрим аналитическое векторное поле (росток векторного поля) в окрестности точки нуль на плоскости, которое определяет динамическую систему

$$
\dot{x}=X(x, y), \quad \dot{y}=Y(x, y) .
$$

ОПРЕДЕлЕниЕ 1.7. 1) Рассмотрим тейлоровские разложения

$$
y X(x, y)=\sum_{i+j=1}^{\infty} a_{i j} x^{i} y^{j}, \quad x Y(x, y)=\sum_{i+j=1}^{\infty} b_{i j} x^{i} y^{j} .
$$

Носителем системы (1.1), а также соответствующего ей векторного поля называется множество таких пар $(i, j)$, что $\left(a_{i j}, b_{i j}\right) \neq(0,0)$. Вектор $\left(a_{i j}, b_{i j}\right)$ называется векторным коэффициентом точки носителя $(i, j)$. Показателем точки носителя $(i, j)$ называется величина

$$
\begin{cases}b_{i j} / a_{i j}, & \text { если } a_{i j} \neq 0, \\ \infty, & \text { если } a_{i j}=0 .\end{cases}
$$

2) Рассмотрим множество

$$
\bigcup_{(i, j)}\left\{(i, j)+\mathbb{R}_{+}^{2}\right\}
$$


где $\mathbb{R}_{+}^{2}$ - положительный квадрант, а объединение берется по всем точкам $(i, j)$, принадлежащим носителю. Граница выпуклой оболочки этого множества состоит из двух открытых лучей и ломаной, которая может состоять и из одной точки. Эта ломаная называется диаграммой Нъютона системы (1.1), а также соответствующего ей векторного поля. Звенья ломаной называются ребрами диаграммы Ньютона, а их концы - ее вершинами.

3) Если граница выпуклой оболочки множества (1.3) содержит луч, который не лежит на координатной оси, то будем называть его неограниченным ребром диаграммы Ньютона.

4) Если вершина диаграммы Ньютона не лежит ни на одной координатной оси, то она называется внутренней.

5) Показателем ребра диаграммы Ньютона называется положительное рациональное число, равное тангенсу угла между ребром и осью ординат.

Если диаграмма Ньютона имеет неограниченное горизонтальное ребро, то припишем ему показатель $\infty$, а если вертикальное, то - 0 .

6) Пусть $\alpha=m / n$ - несократимая дробь. Ребро диаграммы Ньютона с показателем $\alpha$ назовем четным, если одно из чисел $m$ и $n$ четно, и нечетным в противном случае.

7) Рассмотрим ребро $\ell$ диаграммы Ньютона системы (1.1) с показателем $\alpha=m / n$, где $m / n$ - несократимая дробь. Члены разложения (1.2) сгруппируем таким образом, что

$$
y X(x, y)=\sum_{k=0}^{\infty} X_{k}(x, y), \quad x Y(x, y)=\sum_{k=0}^{\infty} Y_{k}(x, y),
$$

где

$$
X_{k}(x, y)=\sum_{n i+m j=k+k_{0}} a_{i j} x^{i} y^{j}, \quad Y_{k}(x, y)=\sum_{n i+m j=k+k_{0}} b_{i j} x^{i} y^{j}
$$

- квазиоднородные полиномы степени $k+k_{0}$ с весами $n$ и $m$ переменных $x$ и $y$ соответственно, $k_{0}>0$. Обозначим

$$
F_{k}(x, y)=n Y_{k}(x, y)-m X_{k}(x, y), \quad k=0,1 .
$$

Положим

$$
\begin{array}{ll}
\Phi_{0}=\frac{X_{0}}{F_{0}}, & \Phi_{1}=\frac{F_{0} X_{1}-F_{1} X_{0}}{F_{0}^{2}} ; \\
\Psi_{0}=-\frac{Y_{0}}{F_{0}}, & \Psi_{1}=\frac{F_{1} Y_{0}-F_{0} Y_{1}}{F_{0}^{2}}=-\alpha \Phi_{1} .
\end{array}
$$

В [16] доказано, что для любого квазиоднородного полинома $R(x, y)$ с весами $n$ и $m(m / n$ - несократимая дробь) переменных $x$ и $y$ справедливо разложение

$$
R(x, y)=A x^{s_{1}} y^{s_{2}} \prod_{i}\left(y^{n}-b_{i} x^{m}\right)^{k_{i}}
$$

где $b_{i}$ - различные ненулевые комплексные числа, $k_{i} \geqslant 0, s_{1} \geqslant 0, s_{2} \geqslant 0$. Многочлен вида $y^{n}-b_{i} x^{m}, b_{i} \neq 0$, называется простым делителем полинома $R(x, y)$. 
8) Ребро $\ell$ диаграммы Ньютона Г называется невырожденным, если многочлен $F_{0}(x, y)$ не имеет вещественных простых делителей. В противном случае ребро называется вырожденным.

9) Пусть $\ell$ и $\tilde{\ell}$ - два примыкающих к внутренней вершине $c$ сверху и снизу ребра (или неограниченных ребра) с показателями $\alpha$ и $\widetilde{\alpha}(0 \leqslant \alpha<\widetilde{\alpha} \leqslant \infty)$, $\beta$ - показатель вершины $c$. Вершина $c$ называется невырожденной, если $\beta \neq \alpha$, $\beta \neq \widetilde{\alpha}$. Вершина $c$ называется вырожденной вверx, если $\beta=\alpha$, и вырожденной вниз, если $\beta=\widetilde{\alpha}$.

10) Для каждой внутренней вершины $c$ диаграммы Ньютона векторного поля $V$ определим две величины $k_{c}$ и $\widetilde{k}_{c}$ следующим образом. Пусть $\ell$ и $\tilde{\ell}$ - два примыкающих к этой вершине сверху и снизу ребра (или неограниченных ребра) с показателями $\alpha$ и $\widetilde{\alpha}(0 \leqslant \alpha<\widetilde{\alpha} \leqslant \infty$.) Занумеруем целочисленные точки на ребре (или неограниченном ребре) $\ell$, присваивая вершине $c$ номер нуль. Через $k_{c}$ обозначим номер ближайшей к вершине $c$ целочисленной точки, лежащей на $\ell$, которая является точкой носителя и показатель $\beta$ которой отличен от $\alpha$. Если такой точки не существует, то положим $k_{c}=\infty$.

Аналогично, перенумеруем целочисленные точки на ребре (или неограниченном ребре) $\widetilde{\ell}$, присваивая вершине $c$ номер нуль. Через $\widetilde{k}_{c}$ обозначим номер ближайшей к вершине $c$ целочисленной точки, лежащей на $\widetilde{\ell}$, которая является точкой носителя и показатель $\widetilde{\beta}$ которой отличен от $\widetilde{\alpha}$. Если такой точки не существует, то положим $\widetilde{k}_{c}=\infty$.

1.6. Раздутие по диаграмме Ньютона. В статьях [16]-[18], [4] описан процесс раздутия, связанный с диаграммой Ньютона. Близкие схемы даны, например, в [19]-[21]. Первый шаг этого процесса состоит в следующем. Окрестность нуля в первом квадранте разбивается на криволинейные секторы, соответствующие ребрам и вершинам диаграммы Ньютона, каждый сектор с помощью степенной замены переменных превращается в прямоугольник. Границы прямоугольников склеиваются с помощью функций перехода.

Векторное поле, полученное после степенной замены переменных и определенное в прямоугольнике, соответствующем ребру диаграммы Ньютона, имеет, вообще говоря, более простые особые точки, а векторное поле, определенное в прямоугольнике, соответствующем внутренней вершине, всегда имеет единственную особую точку, притом элементарную.

Особая точка векторного поля называется элементарной, если матрица линейной части векторного поля в этой особой точке имеет по меньшей мере одно ненулевое собственное значение. Векторное поле в окрестности элементарной особой точки может быть приведено к полиномиальной нормальной форме с помощью гладкой замены переменных. Список нормальных форм приведен в [2; гл. $5, \S 2]$. С точки зрения топологии локального фазового портрета элементарная особая точка может быть седлом, узлом, фокусом, центром и седло-узлом.

Рассмотрим векторное поле с вещественно-изолированной особой точкой и диаграммой Ньютона Г. Пусть $c$ - вершина диаграммы Ньютона $\Gamma$, к которой примыкают два (возможно, неограниченных) ребра $\ell$ и $\tilde{\ell}$ с показателями $\alpha$ и $\widetilde{\alpha}$, где $\widetilde{\alpha}>\alpha, \alpha=m / n, \widetilde{\alpha}=\widetilde{m} / \widetilde{n}-$ несократимые дроби. При этом для 
вертикального неограниченного ребра будем считать, что $m=0, n=1$, а для горизонтального неограниченного $\widetilde{m}=1, \widetilde{n}=0$. Вершине $c$ поставим в соответствие число $d_{c}=n \tilde{m}-m \tilde{n}>0$.

Опишем один шаг процесса раздутия. Пусть $\ell$ - ребро диаграммы Ньютона $\Gamma$ с показателем $\alpha=m / n, m / n-$ несократимая дробь, $\bar{c}$ и $c$ - верхняя и нижняя вершины ребра $\ell$. Предположим, что $c-$ внутренняя вершина. В положительном квадранте $x>0, y>0$ рассмотрим замену переменной

$$
x=z^{n}, \quad y=z^{m} w
$$

и поставим ее в соответствие ребру $\ell$. Пусть $\varepsilon, \delta, \varepsilon_{1}$ - малые положительные числа. Образом при отображении (1.5) прямоугольника

$$
P_{\ell}=\left\{(z, w): \delta^{d_{c}} \leqslant w \leqslant \varepsilon^{-d_{\bar{c}}}, 0 \leqslant z \leqslant \varepsilon_{1}\right\},
$$

который мы поставим в соответствие ребру $\ell$, является некоторый криволинейный сектор $S_{\ell}$ с вершиной в начале координат (см. рис. 2).

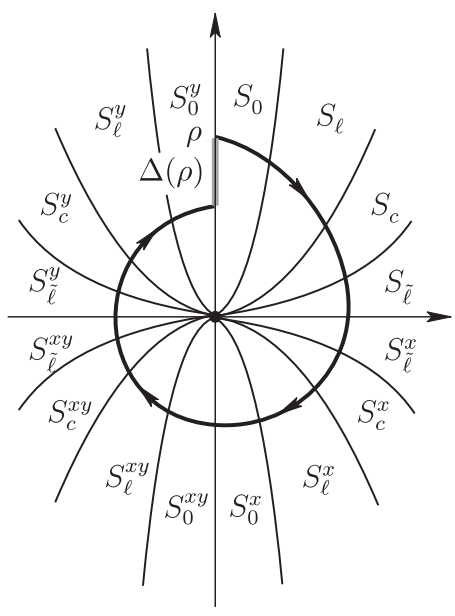

a)

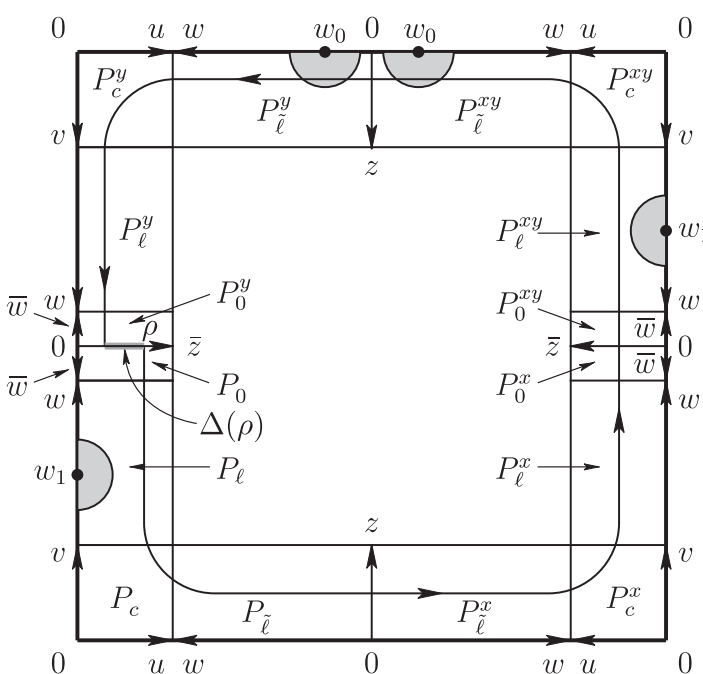

b)

Рис. 2. а) Окрестность монодромной особой точки, $\Delta(\rho)$ - преобразование монодромии. b) Образ окрестности после раздутия, $w_{0}$ и $w_{1}-$ особые точки, полученные в результате раздутия.

Векторное поле, полученное в результате замены (1.5) и деления на некоторую степень $z$, определено в прямоугольнике $P_{\ell}$ и задает систему

$$
\begin{aligned}
\dot{z} & =z X_{0}(1, w)+z^{2}(\ldots), \\
\dot{w} & =w F_{0}(1, w)+z(\ldots) .
\end{aligned}
$$

Особые точки этого векторного поля, расположенные на стороне $z=0$ прямоугольника $P_{\ell}$, ищутся из уравнения $F_{0}(1, w)=0$. 
В случае, если $\ell$ - нижнее ребро диаграммы $Г$ (т. е. $c$ - граничная), в определении $P_{\ell}$ положим $\delta=0$. В этом случае граница сектора $S_{\ell}$ содержит отрезок оси $x$.

Пусть $\ell$ и $\widetilde{\ell}$ - два ребра диаграммы Г с общей вершиной $c$. В малой окрестности нуля между секторами $S_{\ell}$ и $S_{\widetilde{\ell}}$ имеется “зазор” в виде некоторого криволинейного сектора $S_{c}$. Замена переменной

$$
x=u^{n} v^{\widetilde{n}}, \quad y=u^{m} v^{\widetilde{m}}
$$

отображает прямоугольник $P_{c}=\left\{0 \leqslant u \leqslant \varepsilon^{\widetilde{n}}: 0 \leqslant v \leqslant \delta^{n}\right\}$ в сектор $S_{c}$, который мы поставим в соответствие вершине $c$ (см. рис. 2).

Степенной замене (1.7) соответствует матрица показателей $C_{c}=\left(\begin{array}{cc}n & \widetilde{n} \\ m & \tilde{m}\end{array}\right)$. Как показано в [19], [16], степенная замена переменных с матрицей показателей $C$ сопровождается линейным преобразованием носителя с матрицей $C^{t}$. Преобразование с матрицей $C_{c}^{t}$ превращает ребро $\ell$ в вертикальный отрезок, а ребро $\tilde{\ell}$ - в горизонтальный. Поделим векторное поле, полученное в результате замены (1.7), на такие степени $u, v$, чтобы образ вершины $c$ при отображении $C_{c}^{t}$ попал в точку $(1,1)$. Диаграмма Ньютона полученного векторного поля состоит из одной точки $(1,1)$. Набор собственных значений матрицы линейной части векторного поля в этой точке равен векторному коэффициенту точки $(1,1)$ и получается из векторного коэффициента вершины $c$ посредством линейного невырожденного преобразования $C_{c}^{-1}$ [19], [16], а значит, собственные значения не равны нулю одновременно. Таким образом, точка $(u, v)=(0,0)$ в прямоугольнике $P_{c}$ является элементарной.

В случае, если $\ell$ - верхнее ребро диаграммы $\Gamma$, заметим, что при любом $\varepsilon>0$ между $S_{\ell}$ и осью $y$ имеется "зазор" в виде криволинейного сектора $S_{0}$, одной из границ которого является ось ординат.

Замена переменных

$$
x=\bar{w} \bar{z}^{n}, \quad y=\bar{z}^{m}
$$

отображает прямоугольник $P_{0}=\left\{0 \leqslant \bar{w} \leqslant \varepsilon, 0 \leqslant \bar{z} \leqslant \sigma_{1}\right\}$, который мы поставим в соответствие верхней вершине диаграммы Ньютона $\Gamma$, в сектор $S_{0}$.

Параметры $\varepsilon, \varepsilon_{1}, \delta, \sigma_{1}$, участвующие в определениях прямоугольников, берутся настолько малыми, чтобы соответствующие секторы помещались в малую окрестность начала координат, в которой определено исходное векторное поле.

Все замены (1.5)-(1.8) определены в первом квадранте. Чтобы исследовать векторное поле в другом квадранте, его отражают в первый квадрант и проделывают такую же процедуру. Точнее, в первом квадранте кроме векторного поля $V$ рассматриваются отраженные векторные поля $V^{x}, V^{y}, V^{x y}$, которые получаются из векторного поля $V$ соответственно с помощью отражений относительно осей $x, y$ и последовательно $x, y$. Таким образом, после раздутия каждому ребру и каждой вершине диаграммы Ньютона соответствуют 4 прямоугольника. 
Тем самым, окрестность исходной особой точки в результате раздутия заменяется склеенной цепочкой прямоугольников, соответствующих ребрам и вершинам (см. рис. 2). При отображении, обратном раздутию, стороны $z=0$ прямоугольников, соответствующих ребрам, и стороны $u=0$ и $v=0$ (а также $\bar{z}=0)$ прямоугольников, соответствующих вершинам, отображаются в исходную особую точку.

Особые точки, полученные после раздутия и расположенные в прямоугольниках, соответствующих ребрам, появляются парами: в каких-либо двух из четырех прямоугольников, соответствующих ребру. Объединение полуокрестностей этих двух особых точек можно считать одной полной окрестностью одной особой точки (см. рис. 2 , b)). Такие спаренные особые точки находятся во взаимно однозначном соответствии с простыми делителями многочлена $F_{0}(x, y)$.

Поместим начало координат в такую особую точку, если она неэлементарна, и построим диаграмму Ньютона соответствующего векторного поля. Тем самым процесс раздутия продолжается по индукции. Условимся не продолжать процесс раздутия в прямоугольнике $P_{\ell}$, если либо все особые точки, полученные в результате раздутия в прямоугольниках, соответствующих ребру $\ell$, элементарны, либо $F_{0} \equiv 0$. Как показано в [4], процесс раздутия заканчивается на конечном шаге.

1.7. Простейшие монодромные классы. Пусть $V_{0}$ - монодромный росток. Процесс раздутия ростка $V_{0}$ сопроводим построением схемы диаграмм. Изобразим диаграмму Ньютона ростка $V_{0}$. Пусть $\ell$ - любое вырожденное ребро диаграммы Ньютона $\Gamma_{0}$, причем $F_{0} \not \equiv 0$. Это значит, что в процессе раздутия появляется некоторое количество особых точек в прямоугольниках, соответствующих ребру $\ell$. Поскольку $V_{0}-$ монодромный росток, все эти особые точки неэлементарны. Если бы это было не так, то существовала бы характеристическая траектория, входящая в элементарную особую точку и не совпадающая с осью $w$, а ее образ при отображении, обратном раздутию, был бы характеристической траекторией исходного векторного поля $V_{0}$.

Перенумеруем эти особые точки каким-то определенным образом. На рисунке от ребра $\ell$ проведем стрелки вниз соответственно количеству особых точек слева направо в том порядке, в котором они занумерованы. Под стрелкой, соответствующей каждой особой точке, изобразим диаграмму Ньютона ростка векторного поля, полученного после раздутия, в этой особой точке. Проделаем эту процедуру для каждого вырожденного ребра диаграммы ростка $V_{0}$. Далее процесс продолжается по индукции и заканчивается на конечном шаге. Схема диаграмм построена.

Разметим построенную схему диаграмм следующим образом. Каждой внутренней вершине $c$ каждой диаграммы, участвующей в схеме диаграмм, присвоим метку по следующему правилу. Если вершина $c$ невырождена, пометим ее числом 1, если она вырождена вверх (соответственно вниз), то поставим ей в соответствие метку $1+k_{c}$ (соответственно $\left.1 /\left(1+\widetilde{k}_{c}\right)\right)$. Напомним, что если вершина вырождена вверх, то $k_{c} \neq 0$, а если вершина вырождена вниз, то $\widetilde{k}_{c} \neq 0$. 
ОПРЕДЕЛЕНИЕ 1.8. Простейшим монодромным классом называется класс эквивалентности монодромных ростков: два монодромных ростка эквивалентны, если они имеют одинаковую размеченную схему диаграмм.

ОПРЕДЕЛЕНИЕ 1.9. Концевыми диаграммами схемы диаграмм будем называть такие, от ребер которых не идет вниз ни одна стрелка.

ПредлоЖЕНИЕ 1.1. Простейшие монодромные классы являются полуалгебраическими множествами.

ДокАзАтЕльство. Как уже отмечалось выше, при степенной замене с матрицей показателей $C$ носитель векторного поля претерпевает линейное преобразование с матрицей $C^{t}$, а векторные коэффициенты точек носителя - линейное преобразование с матрицей $C^{-1}$. Далее, если $w_{0}$ - особая точка векторного поля $V_{\ell}$, полученного в результате раздутия и определенного в прямоугольнике $P_{\ell}$, и осуществляется сдвиг в эту особую точку, то тейлоровские коэффициенты векторного поля после сдвига являются полиномами от коэффициентов Тейлора поля $V_{\ell}$ и величины $w_{0}$, которая является корнем полинома, коэффициентами которого являются тейлоровские коэффициенты поля $V_{\ell}$.

Зафиксируем размеченную схему диаграмм. Росток с данной размеченной схемой диаграмм является монодромным тогда и только тогда, когда:

1) диаграмма Ньютона ростка $V_{0}$ имеет по одной вершине на каждой координатной оси (если верхняя (нижняя) вершина диаграммы Ньютона является внутренней, то обязательно имеется характеристическая траектория $x=0$ $(y=0))$

2) все остальные диаграммы Ньютона из схемы диаграмм имеют ровно одну вершину на каждой координатной оси (одна из крайних вершин диаграммы Ньютона, полученной после раздутия, является внутренней, так как всегда присутствует характеристическая траектория $z=0$, вторая должна быть граничной по причине п. 1));

3) все ребра всех концевых диаграмм невырожденные (если имеется элементарная особая точка на границе прямоугольника $P_{\ell}$, то помимо направления входа $z=0$ она обязана иметь еще одно);

4) для каждой внутренней вершины каждой диаграммы Ньютона из схемы диаграмм выполняется условие: особая точка, полученная после раздутия в прямоугольнике, соответствующем этой вершине, является седловой.

Все эти условия, а также условия, фиксирующие данную схему диаграмм, задаются алгебраическими уравнениями и неравенствами на тейлоровские коэффициенты всех ростков, участвующих в схеме диаграмм. Таким образом, совокупность этих условий выделяет полуалгебраическое множество в пространстве, координатами в котором являются коэффициенты некоторой конечной струи, а также корни полиномов, коэффициентами которых являются полиномы от коэффициентов струи и корней полиномов, коэффициентами которых являются коэффициенты струи, и так конечное число раз. Проекция этого множества на пространство струй и есть простейший монодромный 
класс, соответствующий данной размеченной схеме диаграмм. Согласно теореме Тарского-Зайденберга [22] проекция полуалгебраического множества является полуалгебраическим множеством. Предложение доказано.

ПРЕДЛОЖЕНИЕ 1.2. Множество всех монодромных ростков является счетным объединением простейших монодромных классов.

ДокАЗАТЕЛЬСтво. Простейших монодромных классов - счетное число и любой монодромный росток принадлежит какому-либо простейшему монодромному классу.

1.8. Сложные монодромные особые точки. Монодромные особые точки из трех перечисленных в п. 1.2 классов таковы, что их диаграмма Ньютона (по крайней мере в некоторой системе координат) состоит из одного невырожденного ребра, концы которого лежат на координатных осях. Такие монодромные особые точки исследовались, например, в [19], [23]. В данном случае преобразование монодромии при подходящем выборе полутрансверсали и параметра на ней является аналитической функцией, тейлоровские коэффициенты которой выражаются через интегралы.

Во всех остальных случаях монодромную особую точку векторного поля будем называть сложной.

Ю. С. Ильяшенко [2] предложил алгоритм исследования сложных монодромных особых точек на основе работы А. Дюлака [24]. Суть метода Дюлака в случае монодромной особой точки состоит в следующем. Производится раздутие особенности, и особая точка заменяется монодромным сложным циклом, который содержит только седловые элементарные особые точки. Преобразование монодромии сложного цикла разбивается в композицию аналитических отображений и отображений соответствия для гиперболических секторов элементарных особых точек. Последние отображения, как правило, не являются аналитическими, а имеют более сложную структуру. В результате асимптотический ряд композиции этих отображений имеет вид

$$
\Delta(\rho)=C_{1} \rho^{\nu_{0}}+\sum_{k=2}^{\infty} P_{k}(\ln \rho) \rho^{\nu_{k}},
$$

где $\left\{\nu_{k}\right\}$ - строго монотонно возрастающая последовательность положительных чисел, стремящаяся к бесконечности, $P_{k}-$ многочлены [24], [25].

Величина $\ln C_{1}$ и коэффициенты полиномов $P_{k}$ являются аналогами ляпуновских фокусных величин в случае сложной монодромой особой точки. Алгоритм их вычисления в самом общем виде изложен в [2].

Доказано [2], [26], что при подходящем выборе полутрансверсали преобразование монодромии имеет линейный главный член асимптотики, т. е.

$$
\Delta(\rho)=C_{1} \rho+o(\rho) \quad \text { при } \quad \rho \rightarrow 0, \quad C_{1}>0 .
$$

Неравенство $\ln C_{1} \neq 0$ является достаточным условием того, чтобы особая точка была фокусом. Уравнение $\ln C_{1}=0$ задает множество нейтральных струй. 
Мы будем называть это множество границей устойчивости, если его коразмерность в соответствующем пространстве струй положительна.

В работе [18] вычислена величина $\ln C_{1}$ для монодромной особой точки произвольного аналитического векторного поля. Ответ формулируется в терминах диаграмм Ньютона векторных полей, возникающих в процессе раздутия особенностей.

Из формулы работы [18] вытекает, что уравнение $\ln C_{1}=0$ задает полуалгебраическое множество. Кроме того, из этой же формулы вытекает, что если все ребра всех диаграмм Ньютона, участвующих в схеме диаграмм четные, то $\ln C_{1} \equiv 0$ на всем простейшем монодромном классе, т. е. преобразование монодромии имеет асимптотику $\Delta(\rho)=\rho+o(\rho)$.

Таким образом, чтобы исследовать вопрос об аналитической разрешимости или неразрешимости проблемы устойчивости, необходимо вычислять второй член асимптотики преобразования монодромии.

1.9. Второй член преобразования монодромии. С целью исследования границ устойчивости в классах монодромных ростков был получен ряд формул для коэффициента при втором члене асимптотики преобразования монодромии. В статье [27] рассматривается случай, когда диаграмма Ньютона состоит из двух невырожденных ребер и выполняется условие $\lambda \neq 1$. Величина $\lambda$ равна минус отношению собственных значений невырожденной седловой особой точки, полученной после раздутия. В статье [28] рассматривается аналогичный случай при $\lambda=1$. В статье [29] рассматривается случай одного невырожденного ребра и случай двух невырожденных ребер, когда соединяющая их вершина вырождена.

В работах [27]-[29] преобразование монодромии исследуется с помощью метода работ [24], [2]. Производится раздутие особой точки по диаграмме Ньютона, при этом окрестность особой точки заменяется цепочкой склеенных прямоугольников, соответствующих ребрам и вершинам (см. рис. 2). Тем самым преобразование монодромии разбивается в суперпозицию отображений соответствия для гиперболических секторов седловых особых точек, расположенных в прямоугольниках, соответствующих вершинам, а также отображений соответствия для прямоугольников, соответствующих ребрам. Последние исследуются путем решения уравнений в вариациях. Границы прямоугольников зависят от малых параметров $\varepsilon$ и $\delta$, от которых коэффициенты асимптотического разложения преобразования монодромии не зависят. Окончательная формула для коэффициента при втором члене асимптотики преобразования монодромии получается предельным переходом при $\varepsilon, \delta \rightarrow 0$.

Актуальной задачей в настоящее время является построение алгоритма вычисления коэффициентов асимптотического разложения преобразования монодромии, не включающего операцию предельного перехода.

Ниже мы сформулируем теоремы из [27] и [28] только для тех случаев, которые используются в доказательстве теоремы 1.2 .

Пусть векторное поле $V$ имеет монодромную особую точку $(0,0)$, и пусть его диаграмма Ньютона состоит из двух ребер $\ell$ и $\widetilde{\ell}$ с показателями $\alpha=m / n$ и 
$\widetilde{\alpha}=\widetilde{m} / \widetilde{n}$ (несократимые дроби), $\alpha<\widetilde{\alpha}$, и имеет по одной вершине на каждой координатной оси. Пусть $F_{0}, \Psi_{0}, \Psi_{1}, \Phi_{0}, \Phi_{1}$ определяются формулами (1.4) и соответствуют ребру $\ell$. Аналогичные функции, соответствующие ребру $\tilde{\ell}$, имеют те же обозначения, но с волной.

Через $\mid \overline{\int_{a}^{b} f(\xi) d \xi}$ обозначается интеграл Адамара от функции $f(x)$ (конечная часть несобственного интеграла), $-\infty \leqslant a<b \leqslant+\infty$ [30].

Теорема 1.3 [27]. Пусть диаграмма Нъютона Г векторного поля V с монодромной особой точкой $(0,0)$ состоит из двух четных невырожденных ребер $\ell$ и $\widetilde{\ell}$ с показателями $\alpha=m / n$ и $\widetilde{\alpha}=\widetilde{m} / \widetilde{n}(\widetilde{\alpha}>\alpha), m / n u \widetilde{m} / \widetilde{n}-$ несократимые дроби, и пусть

$$
0<\lambda=\frac{n b-m a}{\widetilde{n} b-\widetilde{m} a}<1,
$$

где $(a, b)$ - векторный коэффициент вершины диаграммы Нъютона, соединяющей ребра $\ell$ u $\widetilde{\ell}$. Тогда преобразование монодромии особой точки $(0,0)$ векторного поля $V$, определенное вблизи нуля на положительной полуоси у с параметром $\rho=y^{1 / m}$, имеет асимптотику вида

$$
\Delta(\rho)=\rho\left(1+C_{2} \rho^{\lambda}+o\left(\rho^{\lambda}\right)\right), \quad \rho \rightarrow 0,
$$

при этом в случае четного $\widetilde{m}$ и при условии $d=\widetilde{m} n-m \widetilde{n}>1$ уравнение $C_{2}=0$ эквивалентно уравнению

$$
\int_{-\infty}^{+\infty} \frac{\widetilde{\Phi}_{1}(1, w)}{w} \exp \int_{0}^{w} \frac{\widetilde{\Phi}_{0}(1, \xi)}{\xi} d \xi d w=0
$$

Случай $\lambda>1$ сводится к случаю $\lambda<1$, если поменять местами $x$ и $y$.

Теорема 1.4 [28]. Пусть диаграмма Нъютона Г векторного поля $V$ с монодромной особой точкой $(0,0)$ состоит из двух четных невырожденных ребер $\ell$ и $\widetilde{\ell}$ с показателями $\alpha=m / n, \widetilde{\alpha}=\widetilde{m} / \widetilde{n}(\widetilde{\alpha}>\alpha), m / n$ u $\widetilde{m} / \widetilde{n}-$ несократимые дроби, и пусть

$$
\lambda=\frac{n b-m a}{\widetilde{n} b-\widetilde{m} a}=1,
$$

где $(a, b)$ - векторный коэфбициент вериины диаграммы Нъютона, соединяющей ребра $\ell$ u $\widetilde{\ell}$. Тогда, если $d=\widetilde{m} n-m \tilde{n}>1$, то преобразование монодромии имеет асимптотику вида

$$
\Delta(\rho)=\rho\left(1+C_{2} \rho+o(\rho)\right)
$$

где в случае, когда $m$ нечетно, а $\widetilde{m}$ четно, уравнение $C_{2}=0$ эквивалентно уравнению

$$
\begin{aligned}
(1+ & \left.\exp \text { v.p. } \int_{+\infty}^{-\infty} \frac{\widetilde{\Phi}_{0}(1, w)}{w} d w\right) \int_{-\infty}^{+\infty} \frac{\Psi_{1}(w, 1)}{w} \exp \overline{\int_{+\infty}^{w} \frac{\Psi_{0}(\xi, 1)}{\xi} d \xi} d w \\
& =\left(1+\text { exp v.p. } \int_{+\infty}^{-\infty} \frac{\Psi_{0}(w, 1)}{w} d w\right) \int_{-\infty}^{+\infty} \frac{\widetilde{\Phi}_{1}(1, w)}{w} \exp \mid \overline{\int_{+\infty}^{w} \frac{\widetilde{\Phi}_{0}(1, \xi)}{\xi} d \xi} d w .
\end{aligned}
$$


Автор благодарит Ю. С. Ильяшенко за постановки задач и внимание к работе.

\section{2. Доказательство теоремы 1.2}

Рассмотрим множество ростков $M \subset W_{0}$, удовлетворяющих следующим условиям:

1) все ростки из $M$ имеют диаграмму Ньютона $\Gamma$, изображенную на рис. 3 , состоящую из двух ребер $\ell$ и $\widetilde{\ell}$ с показателями $\alpha=m / n=1 / 2$ и $\widetilde{\alpha}=\widetilde{m} / \widetilde{n}=2$ соответственно;

2) ребра $\ell$ и $\tilde{\ell}$ невырождены;

3) $\lambda=(n b-m a) /(\widetilde{n} b-\widetilde{m} a)>0$, где $(a, b)-$ векторный коэффициент вершины $c$, соединяющей ребра $\ell$ и $\tilde{\ell}$.

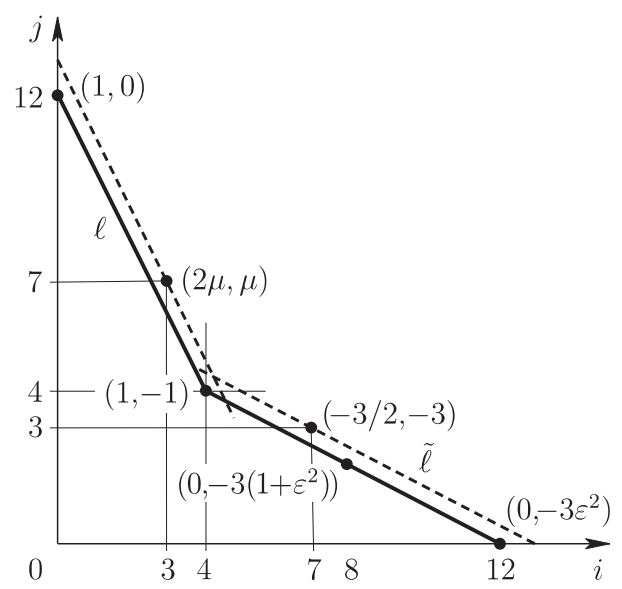

Рис. 3. Диаграмма Ньютона

Согласно определению $1.7,10)$ для вершины $c$ соответствующая величина $k_{c}$ равна нулю. Схема диаграмм состоит из одной диаграммы Г. При такой фиксированной схеме диаграмм условие $\lambda>0$ является необходимым и достаточным условием монодромности, так как величина $\lambda$ равна минус отношению собственных значений седловой особой точки $(u, v)=(0,0)$, расположенной в прямоугольнике $P_{c}$. Отсюда следует, что множество $M$ является простейшим монодромным классом.

Поскольку оба ребра диаграммы Ньютона четные, то преобразование монодромии в классе $M$ имеет тождественный главный член, а коэффициент при втором члене преобразования монодромии задается формулами теоремы 1.3 (при $\lambda \neq 1$ ) и теоремы 1.4 (при $\lambda=1$ ).

Обозначим выражение в левой части (1.9) через $f$. Величина $f$ является функцией от коэффициентов многочленов $\tilde{X}_{0}, \widetilde{Y}_{0}, \widetilde{X}_{1}, \widetilde{Y}_{1}$. Эти коэффициенты являются коэффициентами $k$-струи векторного поля из $M$ при $k \geqslant 11$. Уравнение $f=0$ задает первую границу устойчивости $\gamma_{1}^{1}$ на множестве $M^{(k)} \cap\{\lambda<1\}$, 
поскольку условия $f>0$ и $f<0$ задают множества устойчивых и неустойчивых фокусов соответственно [28], причем, как мы сейчас покажем, codim $\gamma_{1}^{1}=1$ В $M^{(k)}$.

Действительно, зафиксируем любые значения коэффициентов многочленов $\widetilde{X}_{0}, \widetilde{Y}_{0}$, удовлетворяющие условиям принадлежности классу $M$. Тогда $f$ является линейной функцией от коэффициентов многочленов $\widetilde{X}_{1}, \widetilde{Y}_{1}$. Выберем эти коэффициенты так, чтобы выполнялось условие $\widetilde{F}_{1} \equiv 0$. Тогда

$$
f=\int_{-\infty}^{+\infty} \frac{\widetilde{X}_{1}(1, w)}{w \widetilde{F}_{0}(1, w)} \exp \int_{0}^{w} \frac{\widetilde{\Phi}_{0}(1, \xi)}{\xi} d \xi d w
$$

Подставим сюда в общем виде $\widetilde{X}_{1}(1, w)=a_{1} w+a_{2} w^{3}$ и получим, что коэффициент $f$ при $a_{1}$ равен интегралу

$$
\int_{-\infty}^{+\infty} \frac{1}{\widetilde{F}_{0}(1, w)} \exp \int_{0}^{w} \frac{\widetilde{\Phi}_{0}(1, \xi)}{\xi} d \xi d w
$$

который отличен от нуля в силу знакоопределенности $\widetilde{F}_{0}(1, w)$. Таким образом, $\operatorname{grad} f \neq 0$ в некоторых точках множества $M^{(k)}$. Следовательно, $\operatorname{codim} \gamma_{1}^{1}=1$ B $M^{(k)}$.

На множестве $M^{(k)} \cap\{\lambda>1\}$ граница устойчивости $\gamma_{1}^{2}$ строится аналогично. Нужно поменять местами $x$ и $y$ и применить теорему 1.3. В результате такой замены диаграмма Ньютона отражается относительно биссектрисы первого координатного угла, т. е. в нашем случае не меняется. Далее применяем ту же формулу и получаем, что граница $\gamma_{1}^{2}$ также имеет коразмерность один. Множество $\{\lambda=1\}$ также имеет коразмерность один. Поверхности $\gamma_{1}^{1}$ и $\gamma_{1}^{2}$ таковы, что $M^{(k)} \backslash\left(\{\lambda=1\} \cup \gamma_{1}^{1} \cup \gamma_{1}^{2}\right)=J_{01} \cup J_{02}$, где $p_{k}^{-1} J_{0 i} \subset S_{i}, i=1,2$.

Далее построим границу устойчивости коразмерности два.

Согласно теореме 1.1 , при некотором $k \geqslant 11$ существуют полуаналитические в $M^{(k)}$ множества $\gamma_{2}^{1} \subset \gamma_{1}^{1}$ и $\gamma_{2}^{2} \subset \gamma_{1}^{2}, \operatorname{codim} \gamma_{2}^{i} \geqslant 2, i=1,2$, такие, что $\left(\gamma_{1}^{1} \backslash \gamma_{2}^{1}\right) \cup$ $\left(\gamma_{1}^{2} \backslash \gamma_{2}^{2}\right)=J_{11} \cup J_{12}$, где $p_{k}^{-1} J_{1 i} \subset S_{i}, i=1,2$.

Построим границу устойчивости $\gamma_{2}^{0}$ коразмерности два на множестве $M^{(k)} \cap$ $\{\lambda=1\}$. Она задается уравнением (1.10). В уравнении (1.10) перенесем правую часть влево и обозначим через $g$. Зафиксируем любые значения коэффициентов многочленов $X_{0}, Y_{0}, \widetilde{X}_{0}, \widetilde{Y}_{0}$, удовлетворяющие условиям принадлежности классу $M$. Тогда $g$ является линейной функцией от коэффициентов многочленов $X_{1}, Y_{1}, \widetilde{X}_{1}, \widetilde{Y}_{1}$. Выберем эти коэффициенты так, чтобы выполнялись условия $F_{1} \equiv 0, \widetilde{X}_{1} \equiv 0, \widetilde{Y}_{1} \equiv 0$. Тогда уравнение $(1.10)$ эквивалентно уравнению

$$
\int_{-\infty}^{+\infty} \frac{Y_{1}(w, 1)}{w F_{0}(w, 1)} \exp \sqrt{\int_{+\infty}^{w} \frac{\Psi_{0}(\xi, 1)}{\xi} d \xi} d w=0 .
$$

Поделим это уравнение на $\exp \mid \overline{\int_{+\infty}^{0} \frac{\Psi_{0}(\xi, 1)}{\xi} d \xi}$ и подставим в общем виде $Y_{1}(w, 1)=b_{1} w+b_{2} w^{3}$. Тогда коэффициент при $b_{1}$ в левой части равен интегралу

$$
\int_{-\infty}^{+\infty} \frac{1}{F_{0}(w, 1)} \exp \int_{0}^{w} \frac{\Psi_{0}(\xi, 1)}{\xi} d \xi d w
$$


который отличен от нуля в силу знакоопределенности $F_{0}(w, 1)$. Следовательно, градиент функции $g$ отличен от нуля в некоторых точках $M^{(k)}$, а значит, $\operatorname{codim} \gamma_{2}^{0}=2$.

Очевидно, что $\{\lambda=1\} \backslash \gamma_{2}^{0}=J_{10} \cup J_{20}$, где $p_{k}^{-1} J_{i 0} \subset S_{i}, i=1,2$.

В качестве $J_{k}^{\prime}$ возьмем объединение непересекающихся множеств $J_{k}^{\prime}=\gamma_{2}^{0} \cup$ $\gamma_{2}^{1} \cup \gamma_{2}^{2}$. Тогда

$$
M^{(k)} \backslash J_{k}^{\prime}=J_{k}^{1} \cup J_{k}^{2}, \quad \text { где } \quad J_{k}^{1}=J_{01} \cup J_{11} \cup J_{10}, \quad J_{k}^{2}=J_{02} \cup J_{12} \cup J_{20} .
$$

Ниже будет показано, что $J_{k}^{1}, J_{k}^{2}, J_{k}^{\prime}$ непусты.

Граница устойчивости $J_{k}^{\prime}$ является полуаналитическим подмножеством $M^{(k)}$. Покажем, что она не является полуаналитическим подмножеством всего пространства струй $J_{k}^{0}$, т. е. не продолжается аналитически на границу $M^{(k)}$, а именно, $\gamma_{2}^{0}$ не продолжается аналитически на границу $M^{(k)}$. Для этого рассмотрим пересечение $M^{(k)}$ с некоторым линейным многообразием $L$ пространства струй, которое выделяет в $M^{(k)}$ двухпараметрическое семейство струй следующего вида:

$$
\begin{aligned}
& \dot{x}=y^{11}+x^{4} y^{3}+2 \mu x^{3} y^{6}-\frac{3}{2} x^{7} y^{2}, \\
& \dot{y}=-x^{3} y^{4}-3\left(1+\varepsilon^{2}\right) x^{7} y^{2}-3 \varepsilon^{2} x^{11}+\mu x^{2} y^{7}-3 x^{6} y^{3} .
\end{aligned}
$$

Векторные коэффициенты точек носителя для этого семейства прописаны на рис. 3 рядом с соответствующими точками носителя.

Для данного семейства

$$
\begin{gathered}
X_{0}(w, 1)=1+w^{4}, \quad Y_{0}(w, 1)=-w^{4}, \\
F_{0}(w, 1)=2 Y_{0}(w, 1)-X_{0}(w, 1)=-3 w^{4}-1, \quad X_{1}(w, 1)=2 \mu w^{3}, \\
Y_{1}(w, 1)=\mu w^{3}, \quad F_{1}(x, y) \equiv 0, \\
\widetilde{X}_{0}(1, w)=w^{4}, \quad \widetilde{Y}_{0}(1, w)=-w^{4}-3\left(1+\varepsilon^{2}\right) w^{2}-3 \varepsilon^{2}, \\
\widetilde{F}_{0}(1, w)=-3\left(w^{2}+1\right)\left(w^{2}+\varepsilon^{2}\right), \quad \widetilde{X}_{1}(1, w)=-\frac{3}{2} w^{3}, \\
\widetilde{Y}_{1}(1, w)=-3 w^{3}, \quad \widetilde{F}_{1}(x, y) \equiv 0 .
\end{gathered}
$$

Семейство (2.3) удовлетворяет условиям теоремы 1.4 при любом вещественном $\mu$ и ненулевом $\varepsilon$, поэтому граница устойчивости в этом семействе задается уравнением (1.10). Уравнение $\varepsilon=0$ задает границу $M$ в семействе (2.3), поскольку при $\varepsilon=0$ диаграмма Ньютона будет уже не такой, как на рис. 3.

Выпишем уравнение (1.10) для данного семейства (2.3).

Поскольку

$$
\frac{\widetilde{\Phi}_{0}(1, w)}{w}=-\frac{w^{3}}{3\left(w^{2}+1\right)\left(w^{2}+\varepsilon^{2}\right)} \quad \text { и } \quad \frac{\Psi_{0}(w, 1)}{w}=-\frac{w^{3}}{3 w^{4}+1}
$$

- нечетные функции, то выражения в круглых скобках в формуле (1.10) равны двойке. 
Поскольку $\Psi_{1}(w, 1)=\mu w^{3} /\left(3 w^{4}+1\right)$, то левая часть $(1.10)$ равна $\mu K_{0}$, где $K_{0}$ - интеграл от положительной функции, а значит, положительная константа.

Правая часть (1.10) равна

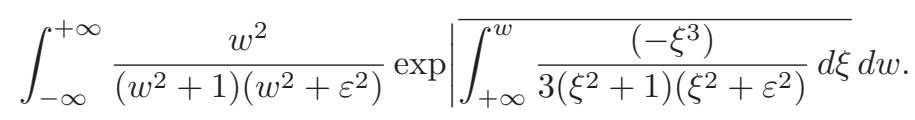

Поскольку $\widetilde{\Phi}_{0}(1, \xi) / \xi=-1 /(3 \xi)+\Psi(\xi)$, где $\Psi(\xi)=O\left(1 / \xi^{2}\right)$ при $\xi \rightarrow \infty$, то при $K>0$ справедливо равенство

$$
\begin{aligned}
\int_{K}^{w} \frac{-\xi^{3}}{3\left(\xi^{2}+1\right)\left(\xi^{2}+\varepsilon^{2}\right)} d \xi= & \int_{K}^{1}\left(-\frac{1}{3 \xi}+\Psi(\xi)\right) d \xi+\int_{1}^{w} \frac{\left(-\xi^{3}\right)}{3\left(\xi^{2}+1\right)\left(\xi^{2}+\varepsilon^{2}\right)} d \xi \\
= & \frac{1}{3} \ln K+\int_{K}^{1}\left(\frac{1}{3 \xi}-\frac{\xi^{3}}{3\left(\xi^{2}+1\right)\left(\xi^{2}+\varepsilon^{2}\right)}\right) d \xi \\
& -\int_{1}^{w} \frac{\xi^{3}}{3\left(\xi^{2}+1\right)\left(\xi^{2}+\varepsilon^{2}\right)} d \xi
\end{aligned}
$$

Вычитая бесконечное слагаемое $(\ln K) / 3$ и переходя к пределу при $K \rightarrow+\infty$, получаем

$$
\begin{aligned}
\overline{\int_{+\infty}^{w} \frac{-\xi^{3}}{3\left(\xi^{2}+1\right)\left(\xi^{2}+\varepsilon^{2}\right)} d \xi}= & \int_{+\infty}^{1}\left(\frac{1}{3 \xi}-\frac{\xi^{3}}{3\left(\xi^{2}+1\right)\left(\xi^{2}+\varepsilon^{2}\right)}\right) d \xi \\
& -\int_{1}^{w} \frac{\xi^{3}}{3\left(\xi^{2}+1\right)\left(\xi^{2}+\varepsilon^{2}\right)} d \xi \\
= & -\frac{1}{6\left(1-\varepsilon^{2}\right)} \ln \left(1+w^{2}\right)+\frac{\varepsilon^{2}}{6\left(1-\varepsilon^{2}\right)} \ln \left(w^{2}+\varepsilon^{2}\right) .
\end{aligned}
$$

Потенцируя и подставляя это выражение в (2.4), получаем, что (1.10) имеет вид $\mu K_{0}=2 I(\varepsilon)$, где

$$
I(\varepsilon)=\int_{0}^{+\infty} \frac{w^{2} d w}{\left(w^{2}+1\right)^{7 / 6+\gamma}\left(w^{2}+\varepsilon^{2}\right)^{1-\gamma}}, \quad \gamma=\gamma(\varepsilon)=\frac{\varepsilon^{2}}{6\left(1-\varepsilon^{2}\right)} .
$$

Неаналитичность границы устойчивости $J_{k}^{\prime}$ вытекает из следующей леммы.

Лемма 2.1. Имеет место соотношение

$$
I(\varepsilon)=A-\frac{\pi}{2} \varepsilon+B \varepsilon^{2}-\frac{\pi}{6} \varepsilon^{3} \ln \varepsilon+O\left(\varepsilon^{3}\right),
$$

где $A$ и В - вещественные числа.

Лемма 2.1 доказывается в следующем разделе. 


\section{3. Асимптотика границы устойчивости}

Интеграл $I(\varepsilon)$ разобьем в сумму интегралов $I(\varepsilon)=I_{1}(\varepsilon)+I_{2}(\varepsilon)$, где

$$
\begin{aligned}
& I_{1}(\varepsilon)=\int_{0}^{1} \frac{w^{2} d w}{\left(w^{2}+1\right)^{7 / 6+\gamma}\left(w^{2}+\varepsilon^{2}\right)^{1-\gamma}}, \\
& I_{2}(\varepsilon)=\int_{1}^{+\infty} \frac{w^{2} d w}{\left(w^{2}+1\right)^{7 / 6+\gamma}\left(w^{2}+\varepsilon^{2}\right)^{1-\gamma}} .
\end{aligned}
$$

Построим асимптотическое разложение $I_{1}(\varepsilon)$. Пользуясь разложением по формуле Тейлора с остаточным членом в форме Лагранжа:

$$
\frac{1}{\left(w^{2}+1\right)^{\gamma}}=1-\ln \left(w^{2}+1\right) \gamma+\frac{1}{2} \frac{\ln ^{2}\left(w^{2}+1\right)}{\left(w^{2}+1\right)} \gamma^{2}, \quad 0<\widetilde{\gamma}<\gamma, \quad \widetilde{\gamma}=\widetilde{\gamma}(w, \gamma),
$$

получаем представление

$$
I_{1}(\varepsilon)=I_{1}^{0}-\gamma I_{1}^{1}+\frac{\gamma^{2}}{2} I_{1}^{2}
$$

где

$$
\begin{gathered}
I_{1}^{0}=\int_{0}^{1} \frac{w^{2} d w}{\left(w^{2}+1\right)^{7 / 6}\left(w^{2}+\varepsilon^{2}\right)^{1-\gamma}}, \quad I_{1}^{1}=\int_{0}^{1} \frac{w^{2} \ln \left(w^{2}+1\right) d w}{\left(w^{2}+1\right)^{7 / 6}\left(w^{2}+\varepsilon^{2}\right)^{1-\gamma}}, \\
I_{1}^{2}=\int_{0}^{1} \frac{w^{2} \ln ^{2}\left(w^{2}+1\right) d w}{\left(w^{2}+1\right)^{7 / 6+\widetilde{\gamma}}\left(w^{2}+\varepsilon^{2}\right)^{1-\gamma}} .
\end{gathered}
$$

Поскольку интеграл $I_{1}^{2}$ ограничен числом $\ln ^{2} 2$, то третье слагаемое в $I_{1}(\varepsilon)$ есть $O\left(\varepsilon^{4}\right)$.

Найдем асимптотические разложения для $I_{1}^{0}$ и $I_{1}^{1}$. При этом будем использовать нижеследующие разложения (3.2), (3.3), а также (3.5) и (3.6):

$$
\begin{gathered}
\left(w^{2}+1\right)^{-7 / 6}=1-\frac{7}{6} w^{2}+w^{4} g_{1}(w), \\
\frac{\ln \left(w^{2}+1\right)}{\left(w^{2}+1\right)^{7 / 6}}=w^{2} g_{2}(w),
\end{gathered}
$$

где $g_{1}(w)$ и $g_{2}(w)$ ограничены на отрезке $[0,1]$.

Далее, построим асимптотику функции $\left(w^{2}+\varepsilon^{2}\right)^{\gamma-1}$ по $\varepsilon$ при $w \neq 0$. Рассмотрим функцию $h(c)=\left(w^{2}+c\right)^{\gamma-1}$, где $\gamma=c /(6(1-c))$. Для этой функции при фиксированном $w \neq 0$ вычислим первую и вторую производные по $c$ :

$$
\begin{aligned}
h^{\prime}(c) & =\left(w^{2}+c\right)^{\gamma-1}\left[\gamma^{\prime}(c) \ln \left(w^{2}+c\right)+(\gamma-1) \frac{1}{w^{2}+c}\right] \\
h^{\prime}(0) & =w^{-2}\left[\frac{1}{6} \ln w^{2}-\frac{1}{w^{2}}\right] \\
h^{\prime \prime}(c) & =\alpha_{0}(c) \frac{\ln \left(w^{2}+c\right)}{\left(w^{2}+c\right)^{1-\gamma}}+\alpha_{1}(c) \frac{\ln ^{2}\left(w^{2}+c\right)}{\left(w^{2}+c\right)^{1-\gamma}}+\alpha_{2}(c) \frac{\ln \left(w^{2}+c\right)}{\left(w^{2}+c\right)^{2-\gamma}} \\
& +\alpha_{3}(c) \frac{1}{\left(w^{2}+c\right)^{2-\gamma}}+\alpha_{4}(c) \frac{1}{\left(w^{2}+c\right)^{3-\gamma}},
\end{aligned}
$$

где $\alpha_{i}(c)$ ограничены при $c \rightarrow 0$. 
Пользуясь двумя последними формулами и формулой Тейлора с остаточным членом в форме Лагранжа, получаем следующие разложения:

$$
\left(w^{2}+\varepsilon^{2}\right)^{\gamma-1}=\frac{1}{w^{2}}+\left(\beta_{1}(\widetilde{\varepsilon}) \frac{\ln \left(w^{2}+\widetilde{\varepsilon}^{2}\right)}{\left(w^{2}+\widetilde{\varepsilon}^{2}\right)^{1-\gamma}}+\beta_{2}(\widetilde{\varepsilon}) \frac{1}{\left(w^{2}+\widetilde{\varepsilon}^{2}\right)^{2-\gamma}}\right) \varepsilon^{2}, \quad \widetilde{\varepsilon}=\widetilde{\varepsilon}(\varepsilon, w),
$$

где $0<\widetilde{\varepsilon}<\varepsilon$ и $\beta_{1}(\widetilde{\varepsilon}), \beta_{2}(\widetilde{\varepsilon})$ ограничены в нуле;

$$
\left(w^{2}+\varepsilon^{2}\right)^{\gamma-1}=\frac{1}{w^{2}}+w^{-2}\left[\frac{1}{6} \ln w^{2}-\frac{1}{w^{2}}\right] \varepsilon^{2}+\frac{1}{2} h^{\prime \prime}\left(\widetilde{\varepsilon}^{2}\right) \varepsilon^{4} .
$$

Подставим (3.2) в выражение для $I_{1}^{0}$, получим

$$
I_{1}^{0}=J_{1}-\frac{7}{6} J_{2}+J_{3}
$$

где

$$
J_{1}=\int_{0}^{1} \frac{w^{2} d w}{\left(w^{2}+\varepsilon^{2}\right)^{1-\gamma}}, \quad J_{2}=\int_{0}^{1} \frac{w^{4} d w}{\left(w^{2}+\varepsilon^{2}\right)^{1-\gamma}}, \quad J_{3}=\int_{0}^{1} \frac{w^{6} g_{1}(w) d w}{\left(w^{2}+\varepsilon^{2}\right)^{1-\gamma}} .
$$

Подставим (3.6) в выражение для $J_{3}$, получим

$$
J_{3}=C_{0}+\left(\frac{1}{6} C_{1}-C_{2}\right) \varepsilon^{2}+\frac{1}{2} R_{1} \varepsilon^{4}
$$

где

$$
\begin{aligned}
& C_{0}= \int_{0}^{1} w^{4} g_{1}(w) d w, \quad C_{1}=\int_{0}^{1} w^{4} \ln w^{2} g_{1}(w) d w, \quad C_{2}=\int_{0}^{1} w^{2} g_{1}(w) d w, \text { (3.9) } \\
& R_{1}=\alpha_{0}\left(\widetilde{\varepsilon}^{2}\right) \int_{0}^{1} \frac{\ln \left(w^{2}+\widetilde{\varepsilon}^{2}\right)}{\left(w^{2}+\widetilde{\varepsilon}^{2}\right)^{1-\gamma}} w^{6} g_{1}(w) d w+\alpha_{1}\left(\widetilde{\varepsilon}^{2}\right) \int_{0}^{1} \frac{\ln ^{2}\left(w^{2}+\widetilde{\varepsilon}^{2}\right)}{\left(w^{2}+\widetilde{\varepsilon}^{2}\right)^{1-\gamma}} w^{6} g_{1}(w) d w \\
& \quad+\alpha_{2}\left(\widetilde{\varepsilon}^{2}\right) \int_{0}^{1} \frac{\ln \left(w^{2}+\widetilde{\varepsilon}^{2}\right)}{\left(w^{2}+\widetilde{\varepsilon}^{2}\right)^{2-\gamma}} w^{6} g_{1}(w) d w \\
& \quad+\alpha_{3}\left(\widetilde{\varepsilon}^{2}\right) \int_{0}^{1} \frac{w^{6} g_{1}(w)}{\left(w^{2}+\widetilde{\varepsilon}^{2}\right)^{2-\gamma}} d w+\alpha_{4}\left(\widetilde{\varepsilon}^{2}\right) \int_{0}^{1} \frac{w^{6} g_{1}(w)}{\left(w^{2}+\widetilde{\varepsilon}^{2}\right)^{3-\gamma}} d w .
\end{aligned}
$$

При $w \in[0,1]$ справедлива оценка

$$
\frac{w^{2 k}}{\left(w^{2}+\widetilde{\varepsilon}^{2}\right)^{k-\gamma}} \leqslant w^{2 \gamma}, \quad k=1,2,3
$$

Оценивая $R_{1}$ по модулю, под интегралами получаем $w^{k}\left|\ln \left(w^{2}+\widetilde{\varepsilon}^{2}\right)\right|, k=2,4$, или $w^{4} \ln ^{2}\left(w^{2}+\widetilde{\varepsilon}^{2}\right)$, или $w^{m}\left|g_{1}(w)\right|, m=0,2$. 
Поскольку

$$
w^{2}\left|\ln \left(w^{2}+\widetilde{\varepsilon}^{2}\right)\right| \leqslant \ln 2,
$$

для $w \in[0,1]$ и $\widetilde{\varepsilon} \in(0,1]$ получаем ограниченность $R_{1}$ при $\varepsilon \rightarrow 0$.

Из (3.1), (3.7) и (3.8) получаем

$$
I_{1}(\varepsilon)=J_{1}-\frac{7}{6} J_{2}-\gamma I_{1}^{1}+C_{0}+\left(\frac{1}{6} C_{1}-C_{2}\right) \varepsilon^{2}+O\left(\varepsilon^{4}\right) .
$$

Теперь исследуем интеграл

$$
I_{1}^{1}=\int_{0}^{1} \frac{w^{2} \ln \left(w^{2}+1\right) d w}{\left(w^{2}+1\right)^{7 / 6}\left(w^{2}+\varepsilon^{2}\right)^{1-\gamma}}
$$

Подставим в $I_{1}^{1}$ сначала (3.3), а затем (3.5). Получим

$$
I_{1}^{1}=\int_{0}^{1} \frac{w^{2} g_{2}(w) d w}{\left(w^{2}+\varepsilon^{2}\right)^{1-\gamma}}=\int_{0}^{1} w^{2} g_{2}(w) d w+\varepsilon^{2} R_{2}
$$

где

$$
R_{2}=\beta_{1}(\widetilde{\varepsilon}) \int_{0}^{1} \frac{\ln \left(w^{2}+\widetilde{\varepsilon}^{2}\right)}{\left(w^{2}+\widetilde{\varepsilon}^{2}\right)^{1-\gamma}} w^{4} g_{2}(w) d w+\beta_{2}(\widetilde{\varepsilon}) \int_{0}^{1} \frac{w^{2} g_{2}(w)}{\left(w^{2}+\widetilde{\varepsilon}^{2}\right)^{2-\gamma}} d w
$$

В силу оценок (3.10) и (3.11) величина $R_{2}$ ограничена при $\varepsilon \rightarrow 0$.

Обозначим $C_{3}=\int_{0}^{1} w^{2} g_{2}(w) d w$. Таким образом, из $(3.12)$ находим

$$
I_{1}(\varepsilon)=J_{1}-\frac{7}{6} J_{2}+C_{0}+\left(\frac{1}{6} C_{1}-C_{2}-\frac{1}{6} C_{3}\right) \varepsilon^{2}+O\left(\varepsilon^{4}\right) .
$$

Найдем асимптотику интеграла

$$
J_{1}=\int_{0}^{1} \frac{w^{2} d w}{\left(w^{2}+\varepsilon^{2}\right)^{1-\gamma}} .
$$

После замены $w=\varepsilon y$ получим, что $J_{1}=\varepsilon^{1-2 \gamma} J_{1}^{\prime}$, где

$$
\begin{gathered}
J_{1}^{\prime}=\int_{0}^{1 / \varepsilon} \frac{y^{2} d y}{\left(y^{2}+1\right)^{1-\gamma}}=K_{1}+K_{2} \\
K_{1}=\int_{0}^{1} \frac{y^{2}\left(y^{2}+1\right)^{\gamma} d y}{\left(y^{2}+1\right)}, \quad K_{2}=\int_{1}^{1 / \varepsilon} \frac{y^{2}\left(y^{2}+1\right)^{\gamma} d y}{\left(y^{2}+1\right)} .
\end{gathered}
$$


Из разложения

$$
\left(1+y^{2}\right)^{\gamma}=1+\gamma\left(1+\widetilde{y}^{2}\right)^{\gamma-1} y^{2}, \quad 0<\widetilde{y}<y
$$

получаем

$$
K_{1}=\int_{0}^{1} \frac{y^{2} d y}{y^{2}+1}+\gamma \int_{0}^{1} \frac{y^{4} d y}{\left(1+y^{2}\right)\left(1+\widetilde{y}^{2}\right)^{1-\gamma}}=1-\frac{\pi}{4}+O\left(\varepsilon^{2}\right) .
$$

Из разложения

$$
\left(1+\frac{1}{y^{2}}\right)^{\gamma}=1+\gamma\left(1+\frac{1}{\widetilde{y}^{2}}\right)^{\gamma-1} \frac{1}{y^{2}}, \quad \widetilde{y}>y,
$$

получаем

$$
K_{2}=\int_{1}^{1 / \varepsilon} \frac{y^{2+2 \gamma}\left(1+1 / y^{2}\right)^{\gamma} d y}{\left(y^{2}+1\right)}=L_{1}+\gamma L_{2}
$$

где

$$
L_{1}=\int_{1}^{1 / \varepsilon} \frac{y^{2+2 \gamma} d y}{\left(y^{2}+1\right)}, \quad L_{2}=\int_{1}^{1 / \varepsilon} \frac{y^{2 \gamma} d y}{\left(y^{2}+1\right)\left(1+1 / \widetilde{y}^{2}\right)^{1-\gamma}}=O(1)
$$

Учитывая разложение $\left(1+1 / y^{2}\right)^{-1}=1-1 / y^{2}+1 /\left(y^{2}\left(1+y^{2}\right)\right)$, находим, что

$$
L_{1}=\int_{1}^{1 / \varepsilon} \frac{y^{2 \gamma} d y}{\left(1+1 / y^{2}\right)}=\frac{\varepsilon^{-2 \gamma-1}-1}{2 \gamma+1}-\frac{\varepsilon^{1-2 \gamma}-1}{2 \gamma-1}+L_{3},
$$

где

$$
L_{3}=\int_{1}^{1 / \varepsilon} \frac{y^{2 \gamma} d y}{y^{2}\left(y^{2}+1\right)} .
$$

В силу разложения $y^{2 \gamma}=1+2 y^{2 \widetilde{\gamma}}(\ln y) \gamma, 0<\widetilde{\gamma}<\gamma$, получаем, что $L_{3}=$ $N_{1}+\gamma N_{2}$, где

$$
\begin{aligned}
& N_{1}=\int_{1}^{1 / \varepsilon} \frac{d y}{y^{2}\left(y^{2}+1\right)}=-\varepsilon-\operatorname{arctg} \frac{1}{\varepsilon}+1+\frac{\pi}{4}=1-\frac{\pi}{4}+O\left(\varepsilon^{3}\right), \\
& N_{2}=\int_{1}^{1 / \varepsilon} \frac{2 y^{2} \widetilde{\gamma} \ln y d y}{y^{2}\left(y^{2}+1\right)}=O(1) .
\end{aligned}
$$

Следовательно, $L_{3}=1-\pi / 4+O\left(\varepsilon^{2}\right)$ и, значит,

$$
\begin{aligned}
& L_{1}=1-\frac{\pi}{4}+\frac{\varepsilon^{-2 \gamma-1}-1}{2 \gamma+1}+\frac{\varepsilon^{1-2 \gamma}-1}{1-2 \gamma}+O\left(\varepsilon^{2}\right), \\
& K_{2}=-1-\frac{\pi}{4}+\frac{\varepsilon^{-2 \gamma-1}}{1+2 \gamma}+\frac{\varepsilon^{1-2 \gamma}}{1-2 \gamma}+O\left(\varepsilon^{2}\right) .
\end{aligned}
$$


Таким образом, из (3.16), (3.18) и (3.14) следует, что

$$
\begin{aligned}
J_{1} & =\varepsilon^{1+2 \gamma}\left(K_{1}+K_{2}\right)=\varepsilon^{1+2 \gamma}\left(-\frac{\pi}{2}+\frac{\varepsilon^{-2 \gamma-1}}{2 \gamma+1}+\frac{\varepsilon^{1-2 \gamma}}{1-2 \gamma}+O\left(\varepsilon^{2}\right)\right) \\
& =-\frac{\pi}{2} \varepsilon^{1+2 \gamma}+\frac{1}{1+2 \gamma}+\frac{\varepsilon^{2}}{1-2 \gamma}+O\left(\varepsilon^{3}\right) .
\end{aligned}
$$

Итак,

$$
J_{1}=1-\frac{\pi}{2} \varepsilon+\frac{2}{3} \varepsilon^{2}-\frac{\pi}{6} \varepsilon^{3} \ln \varepsilon+O\left(\varepsilon^{3}\right)
$$

Найдем теперь асимптотику интеграла

$$
J_{2}=\int_{0}^{1} \frac{w^{4} d w}{\left(w^{2}+\varepsilon^{2}\right)^{1-\gamma}} .
$$

После замены $w=\varepsilon y$ получим, что $J_{2}=\varepsilon^{3+2 \gamma} J_{2}^{\prime}$, где

$$
\begin{aligned}
& J_{2}^{\prime}=\int_{0}^{1 / \varepsilon} \frac{y^{4} d y}{\left(y^{2}+1\right)^{1-\gamma}}=T_{1}+T_{2}, \\
& T_{1}=\int_{0}^{1} \frac{y^{4} d y}{\left(y^{2}+1\right)^{1-\gamma}}=O(1), \\
& T_{2}=\int_{1}^{1 / \varepsilon} \frac{y^{4} d y}{\left(y^{2}+1\right)^{1-\gamma}}=\int_{1}^{1 / \varepsilon} \frac{y^{4+2 \gamma}\left(1+1 / y^{2}\right)^{\gamma} d y}{\left(y^{2}+1\right)} .
\end{aligned}
$$

В силу (3.17)

$$
T_{2}=U_{1}+\gamma U_{2}
$$

где

$$
\begin{gathered}
U_{2}=\int_{1}^{1 / \varepsilon} \frac{y^{2+2 \gamma} d y}{\left(y^{2}+1\right)\left(1+1 / \widetilde{y}^{2}\right)^{1-\gamma}} \leqslant \int_{1}^{1 / \varepsilon} y^{2 \gamma} d y=O\left(\varepsilon^{-1-2 \gamma}\right), \quad \gamma U_{2}=O(1), \\
U_{1}=\int_{1}^{1 / \varepsilon} \frac{y^{4+2 \gamma} d y}{\left(y^{2}+1\right)}=\int_{1}^{1 / \varepsilon} \frac{y^{2+2 \gamma} d y}{\left(1+1 / y^{2}\right)} .
\end{gathered}
$$

В силу разложения $\left(1+1 / y^{2}\right)^{-1}=1-1 / y^{2}+\left(1 / y^{4}\right) g_{4}(y)$, где $g_{4}(y)$ ограничена при $y \geqslant 1$, получаем, что

$$
U_{1}=\int_{1}^{1 / \varepsilon} y^{2+2 \gamma} d y-\int_{1}^{1 / \varepsilon} y^{2 \gamma} d y+\int_{1}^{1 / \varepsilon} \frac{g_{4}(y) d y}{y^{2-2 \gamma}}=\frac{\varepsilon^{-3-2 \gamma}}{3+2 \gamma}-\frac{\varepsilon^{-1-2 \gamma}}{1+2 \gamma}+O(1) .
$$


Отсюда и из $(3.20),(3.21)$ и (3.22) вытекают соотношения

$$
\begin{aligned}
& J_{2}^{\prime}=U_{1}+O(1)=\frac{\varepsilon^{-3-2 \gamma}}{3+2 \gamma}-\frac{\varepsilon^{-1-2 \gamma}}{1+2 \gamma}+O(1), \\
& J_{2}=\varepsilon^{3+2 \gamma} J_{2}^{\prime}=\frac{1}{3+2 \gamma}-\frac{\varepsilon^{2}}{1+2 \gamma}+O\left(\varepsilon^{3}\right)=\frac{1}{3}-\frac{28}{27} \varepsilon^{2}+O\left(\varepsilon^{3}\right) .
\end{aligned}
$$

Подставляя (3.19) и (3.23) в (3.13), получаем

$$
I_{1}(\varepsilon)=\frac{11}{18}+C_{0}-\frac{\pi}{2} \varepsilon+D_{1} \varepsilon^{2}-\frac{\pi}{6} \varepsilon^{3} \ln \varepsilon+O\left(\varepsilon^{3}\right),
$$

где $C_{0}$ определено формулой $(3.9), D_{1}=152 / 81+C_{1} / 6-C_{2}-C_{3} / 6$.

Теперь исследуем асимптотику интеграла

$$
I_{2}(\varepsilon)=\int_{1}^{+\infty} \frac{w^{2} d w}{\left(w^{2}+1\right)^{7 / 6+\gamma}\left(w^{2}+\varepsilon^{2}\right)^{1-\gamma}} .
$$

После замены $w=1 / u$ получаем

$$
I_{2}(\varepsilon)=\int_{0}^{1} \frac{u^{1 / 3}\left(1+\varepsilon^{2} u^{2}\right)^{\gamma} d u}{\left(1+u^{2}\right)^{7 / 6+\gamma}\left(1+\varepsilon^{2} u^{2}\right)}
$$

\section{Обозначим}

$$
h(c)=\frac{u^{1 / 3}\left(1+c u^{2}\right)^{\gamma}}{\left(1+u^{2}\right)^{7 / 6+\gamma}\left(1+c u^{2}\right)}, \quad \text { где } \quad \gamma=\frac{c}{6(1-c)} .
$$

Тогда

$$
h^{\prime \prime}(c)=\frac{u^{1 / 3} Q\left(c, u, \ln \left(1+u^{2}\right), \ln \left(1+c u^{2}\right)\right)}{(1-c)^{4}\left(1+u^{2}\right)^{7 / 6+\gamma}\left(1+c u^{2}\right)^{1-\gamma}},
$$

где $Q$ - полином от четырех переменных; $h^{\prime \prime}(c)$ равномерно ограничена при $u \in[0,1]$ и $c \rightarrow 0$. Раскладывая $h$ по формуле Тейлора до второй степени, получаем

$$
h\left(\varepsilon^{2}\right)=\frac{u^{1 / 3}}{\left(1+u^{2}\right)^{7 / 6}}-\frac{u^{7 / 3}+\frac{1}{6} u^{1 / 3} \ln \left(1+u^{2}\right)}{\left(1+u^{2}\right)^{7 / 6}} \varepsilon^{2}+\frac{1}{2} h^{\prime \prime}\left(\widetilde{\varepsilon}^{2}\right) \varepsilon^{4}, \quad 0<\widetilde{\varepsilon}<\varepsilon .
$$

Отсюда следует, что

$$
I_{2}(\varepsilon)=P_{0}+P_{1} \varepsilon^{2}+O\left(\varepsilon^{4}\right)
$$


где

$$
P_{0}=\int_{0}^{1} \frac{u^{1 / 3}}{\left(1+u^{2}\right)^{7 / 6}} d u, \quad P_{1}=-\int_{0}^{1} \frac{u^{7 / 3}+\frac{1}{6} u^{1 / 3} \ln \left(1+u^{2}\right)}{\left(1+u^{2}\right)^{7 / 6}} d u .
$$

Из (3.24) и (3.25) находим

$$
I(\varepsilon)=I_{1}(\varepsilon)+I_{2}(\varepsilon)=A-\frac{\pi}{2} \varepsilon+B \varepsilon^{2}-\frac{\pi}{6} \varepsilon^{3} \ln \varepsilon+O\left(\varepsilon^{3}\right),
$$

где $A=11 / 18+C_{0}+P_{0}, B=D_{1}+P_{1}$. Лемма 2.1 доказана.

Вычисление коэффициентов. Константа $A$, участвующая в формулировке леммы 2.1, может быть представлена в виде $A=I(0)=\mathrm{B}(1 / 2,2 / 3) / 2 \approx$ 1.29355 , где В $(\cdot, \cdot)$ - бета-функция.

Приближенно вычисляя интегралы, получаем

$$
\begin{aligned}
C_{1} & =\int_{0}^{1} \ln w^{2}\left(\left(1+w^{2}\right)^{-7 / 6}-1+\frac{7}{6} w^{2}\right) d w \approx-0.06718, \\
C_{2} & =\int_{0}^{1}\left(\left(1+w^{2}\right)^{-7 / 6}-1+\frac{7}{6} w^{2}\right) \frac{d w}{w^{2}} \approx 0.26571, \\
C_{3} & =\int_{0}^{1} \frac{\ln \left(1+w^{2}\right) d w}{\left(1+w^{2}\right)^{7 / 6} \approx 0.16157,} \\
P_{1} & =-\int_{0}^{1} \frac{u^{7 / 3}+(1 / 6) u^{1 / 3} \ln \left(1+u^{2}\right)}{\left(1+u^{2}\right)^{7 / 6}} d u \approx-0.19968, \\
D_{1} & :=\frac{152}{81}+\frac{1}{6} C_{1}-C_{2}-\frac{1}{6} C_{3} \approx 1.57271, \\
B & =D_{1}+P_{1} \approx 1.36712 .
\end{aligned}
$$

\section{Список литературы}

[1] В.И. Арнольд, "О локальных задачах анализа", Вестн. Моск. ун-та. Сер. 1. Maтем. Mех., 25:2 (1970), 52-56; англ. пер.: V.I. Arnol'd, "Local problems of analysis", Mosc. Univ. Math. Bull., 25(1970):1-2 (1972), 77-80.

[2] В.И. Арнольд, Ю.С. Ильяшенко, "Обыкновенные дифференциальные уравнения", Динамические системы - 1, Итоги науки и техники. Соврем. проблемы матем. Фундам. напр., 1, ВИНИТИ, М., 1985, 7-140; англ. пер.: V.I. Arnol'd, Yu.S. Ilyashenko, "Ordinary differential equations", Dynamical systems, I, Encyclopaedia Math. Sci., 1, Springer, Berlin, 1988, 1-148.

[3] Ю. С. Ильяшенко, "Алгебраически и аналитически разрешимые локальные задачи теории обыкновенных дифференциальных уравнений”, Тр. сем. им. И. Г. Петровского, 1987, № 12, 118-136; англ. пер.: Yu. S. Il'yashenko, "Algebraically and analytically solvable local problems in the theory of ordinary differential equations", J. Soviet Math., 47:3 (1989), 2570-2584. 
[4] Н. Б. Медведева, "Об аналитической разрешимости проблемы различения центра и фокуса", Нелинейные аналитические дифференииальные уравнения, Сборник статей, Тр. МИАН, 254, Наука, М., 2006, 11-100; англ. пер.: N. В. Medvedeva, "On the analytic solvability of the problem of distinguishing between center and focus", Proc. Steklov Inst. Math., 254:1 (2006), 7-93.

[5] В. И. Арнольд, "Аналитическая неразрешимость проблемы устойчивости по Ляпунову и проблемы топологической классификации особых точек аналитической системы дифференциальных уравнений", Функи. анализ и его прил., 4:3 (1970), 1-9; англ. пер.: V.I. Arnol'd, "Algebraic unsolvability of the problem of Lyapunov stability and the problem of topological classification of singular points of an analytic system of differential equations", Funct. Anal. Appl., 4:3 (1970), 173-180.

[6] Ю.С. Ильяшенко, “Аналитическая неразрешимость проблемы устойчивости и проблемы топологической классификации особых точек аналитических систем дифференциальных уравнений", Матем. сб., 99(141):2 (1976), 162-175; англ. пер.: Yu.S. Il'yashenko, "Analytic unsolvability of the stability problem and the problem of topological classification of the singular points of analytic systems of differential equations", Math. USSR-Sb., 28:2 (1976), 140-152.

[7] Yu. S. Il'yashenko, Finiteness theorems for limit cycles, Transl. Math. Monogr., 94, Amer. Math. Soc., Providence, RI, 1991, x+288 c.

[8] А. Пуанкаре, О кривых, определяемых дифференииальными уравнениями, Гостехиздат, М.-Л., 1947, 392 с.

[9] А. М. Ляпунов, Собрание сочинений, т. 2, Изд-во АН СССР, М.-Л., 1956, 472 с.

[10] А. М. Ляпунов, Общая задача об устойчивости движения, ГИТТЛ, М.-Л., 1950, 471 с.; англ. пер.: А. M. Lyapunov, The general problem of the stability of motion, With a biography of Lyapunov by V. I. Smirnov and a bibliography of Lyapunov's works by J. F. Barrett, ed. A. T. Fuller, Taylor \& Francis, London, 1992, ix+270 pp.

[11] R. Moussu, "Symmétrie et forme normale des centres et foyers dégénérés", Ergodic Theory Dynam. Systems, 2:2 (1982), 241-251.

[12] В. В. Немыцкий, В. В. Степанов, Качественная теория дифференциальных уравнений, ОГИЗ, М.-Л., 1947, 448 с.; англ. пер.: V.V. Nemytskii, V.V. Stepanov, Qualitative theory of differential equations, Princeton Math. Ser., 22, Princeton Univ. Press, Princeton, NJ, 1960, viii+523 pp.

[13] F. Dumortier, "Singularities of vector fields on the plane", J. Differential Equations, 23:1 (1977), 53-106.

[14] Ю.С. Ильяшенко, "Алгебраическая неразрешимость и почти алгебраическая разрешимость проблемы центр-фокус", Функи. анализ и его прил., 6:3 (1972), 30-37; англ. пер.: Yu. S. Il'yashenko, "Algebraic nonsolvability and almost algebraic solvability of the center-focus problem", Funct. Anal. Appl., 6:3 (1972), 197-202.

[15] Н. Б. Медведева, "Об аналитической разрешимости проблемы различения центра и фокуса", Докл. РАН, 394:6 (2004), 735-738; англ. пер.: ․ B. Medvedeva, "On an analytic solvability of the center-focus problem", Dokl. Math., 69:1 (2004), 120-122.

[16] Ф.С. Березовская, Н. Б. Медведева, “Асимптотика преобразования монодромии особой точки с фиксированной диаграммой Ньютона", Тр. сем. им. И.Г. Петровского, 1991, № 15, 156-177; англ. пер.: F. S. Berezovskaya, N. B. Medvedeva, "The asymptotics of the return map of a singular point with fixed Newton diagram", J. Soviet Math., 60:6 (1992), 1765-1781.

[17] Н.Б. Медведева, "Критерий монодромности особой точки векторного поля на плоскости", Алгебра и анализ, 13:2 (2001), 130-150; англ. пер.: N. B. Medvedeva, 
"A monodromy criterion for a singular point of a vector field on the plane", St. Petersburg Math. J., 13:2 (2002), 253-268.

[18] Н. Б. Медведева, Е. В. Мазаева, "Достаточное условие фокуса для монодромной особой точки", Тр. ММО, 63, Изд-во Моск. ун-та, М., 2002, 87-114; англ. пер.: N.B. Medvedeva, E. V. Mazaeva, "A sufficient focus condition for a monodromic singular point", Trans. Moscow Math. Soc., 2002 (2003), 77-103.

[19] А. Д. Брюно, Локалъный метод нелинейного анализа дифференииалъных уравнений, Наука, М., 1979, 253 с.; англ. пер.: А. D. Bruno, Local methods in nonlinear differential equations, Part I. The local method of nonlinear analysis of differential equations. Part II. The sets of analyticity of a normalizing transformation, Springer Ser. Soviet Math., Springer-Verlag, Berlin, 1989, x+348 pp.

[20] Ф.С. Березовская, Сложная стационарная точка системь на плоскости: структура окрестности и индекс, Препринт, ОНТИ НЦБИ, Пущино, 1978, 15 с.

[21] М. Фроммер, "Интегральные кривые обыкновенного дифференциального уравнения первого порядка в окрестности особой точки, имеющей рациональный характер", УМH, 1941, № 9, 212-253; пер. с нем.: M. Frommer, "Die Integralkurven einer gewohnlichen Differentialgleichung erster Ordnung in der Umgebung rationaler Unbestimmtheitsstellen", Math. Ann., 99 (1928), 222-272.

[22] Е.А. Горин, "Об асимптотических свойствах многочленов и алгебраических функций от нескольких переменных", УМН, 16:1(97) (1961), 91-118; англ. пер.: E. A. Gorin, "Asymptotic properties of polynomials and algebraic functions of several variables", Russian Math. Surveys, 16:1 (1961), 93-119.

[23] В. П. Варин, "Отображение последования некоторых полиномиальных систем дифференциальных уравнений”, Матем. сб., 195:7 (2004), 3-20; англ. пер.: V.P. Varin, "Poincaré map for some polynomial systems of differential equations", Sb. Math., 195:7 (2004), 917-934.

[24] А. Дюлак, O предельных ииклах, Наука, М., 1980, 157 с.; пер. с франц.: Н. Dulac, "Sur les cycles limites", Bull. Soc. Math. France, 51 (1923), 45-188.

[25] Ю. С. Ильяшенко, “Мемуар Дюлака 'О предельных циклах' и смежные вопросы локальной теории дифференциальных уравнений”, УМH, 40:6(246) (1985), 41-78; англ. пер.: Yu. S. Il'yashenko, "Dulac's memoir 'On limit cycles' and related problems of the local theory of differential equations", Russian Math. Surveys, 40:6 (1985), $1-49$.

[26] Н. Б. Медведева, "Главный член преобразования монодромии монодромной особой точки линеен", Сиб. матем. журн., 33:2 (1992), 116-124; англ. пер.: N. B. Medvedeva, "Principal term of the monodromy transformation of a monodromic singular point is linear", Siberian Math. J., 33:2 (1992), 280-288.

[27] А.С. Воронин, Н.Б. Медведева, "Асимптотика преобразования монодромии в случае двух четных ребер диаграммы Ньютона", Вестн. Челяб. гос. ун-та. Сер. 3. Матем. Мех. Информ., 27(242):14 (2011), 12-26.

[28] А.С. Воронин, Н.Б. Медведева, "Устойчивость монодромных особых точек с фиксированной диаграммой Ньютона", Вестн. Удмурт. ун-та. Сер. Матем. Мех. Компъютерные науки, 3 (2009), 34-49.

[29] А.С. Воронин, Н.Б. Медведева, "Асимптотика преобразования монодромии в некоторых классах монодромных ростков", Изв. РАН. Сер. матем., 77:2 (2013), 35-52; англ. пер.: A. S. Voronin, N. B. Medvedeva, "Asymptotics of the monodromy transformation in certain classes of monodromy germs", Izv. Math., 77:2 (2013), 253-270. 
[30] Ж. Адамар, Задача Коши для линейных уравнений с частными производными гиперболического типа, Наука, М., 1978, 351 с.; пер. с франц.: J. Hadamard, Le problème de Cauchy et les équations aux derivées partielles linéaires hyperboliques, Hermann, Paris, 1932, 542 pp.

Н. Б. Медведева (N. B. Medvedeva)

Челябинский государственный университет;

Южно-Уральский государственный университет

E-mail: medv@csu.ru
Поступила в редакцию

17.02 .2013 